\title{
Dealing with the Empty Vehicle Movements in Personal Rapid Transit System with Batteries Constraints in a Dynamic Context
}

\author{
Ezzeddine Fatnassi, ${ }^{1}$ Olfa Chebbi, ${ }^{2}$ and Jouhaina Chaouachi ${ }^{3}$ \\ ${ }^{1}$ Institut Superieur de Gestion, University of Tunis, 41 rue de la Liberté, 2000 Le Bardo, Tunisia \\ ${ }^{2}$ Université de Kairouan, Institut Supérieur d'Informatique et de Gestion de Kairouan, Avenue Khemais El Alouini, \\ 3100 Kairouan, Tunisia \\ ${ }^{3}$ Institut des Hautes Etudes Commerciales, Carthage, Tunisia \\ Correspondence should be addressed to Olfa Chebbi; olfaa.chebbi@gmail.com
}

Received 5 September 2016; Revised 5 January 2017; Accepted 8 February 2017; Published 13 April 2017

Academic Editor: Zhi-Chun Li

Copyright ( 2017 Ezzeddine Fatnassi et al. This is an open access article distributed under the Creative Commons Attribution License, which permits unrestricted use, distribution, and reproduction in any medium, provided the original work is properly cited.

\begin{abstract}
The Personal Rapid Transit is a new emergent transportation tool. It relies on using a set of small driverless electric vehicles to transport people on demand. Because of the specific on-demand characteristic of the Personal Rapid Transit system, many Personal Rapid Transit vehicles would move empty which results in a high level of wasted transportation capacity. This is enhanced while using Personal Rapid Transit vehicles with limited electric battery capacity. This paper deals with this problem in a real time context while minimizing the set of empty vehicle movements. First, a mathematical formulation to benchmark waiting time of passengers in Personal Rapid Transit systems is proposed. Then, a simulation model that captures the main features of the Personal Rapid Transit system is developed. A decision support system which integrates several real time solution strategies as well as a simulation module is proposed. Our dispatching strategies are evaluated and compared based on our simulation model. The efficiency of our method is tested through extensive test studies.
\end{abstract}

\section{Introduction}

1.1. Background and Literature Review. PRT could be seen as a sustainable and efficient transportation system that has the potential to overcome issues related to urban mobility. PRT is an on-demand transportation system that uses small driverless electric vehicles. It was introduced first in 1953 and came up to realization recently in airport (Heathrow Airport, London, UK), universities (Morgantown, West Virginia, US), and smart cities (Masdar city, Abu Dhabi, UAE).

According to Cottrell [1], more than 200 research papers related to PRT have been published since 1964. The period from 1971 to 1975 was the most active for PRT research, primarily because the US government financed several reports, such as the studies funded by the Urban Mass Transportation Administration (UMTA) branch of the Department of Housing and Urban Development (HUD) [2]. The US government withdrew its support in 1974, but there was a resurgence of interest in PRT systems in the 90s. This coincided with progress in different PRT components, such as vehicles, rails, and batteries. From 2001 to 2004, research was conducted to test the feasibility of innovative city transport (more details could be found in http://www.transport-research.info/ project/evaluation-and-demonstration-innovative-city-transport) in five European cities (Eindhoven and Almelo in the Netherlands, Cardiff in Wales, Rome Ciampino in Italy, and Huddinge in Sweden). The relevant study assessed the social, economic, technical, and environmental benefits of the PRT system, and its results supported the implementation of these PRT projects.

Several studies related to PRT have focused on the feasibility and design of such a system [3-6] and, until recently, had sought to tackle operating issues in PRT, such as waiting times for passengers $[7,8]$, dynamic routing [9-11], agentbased simulation [12], network design [13], fleet sizing [14], and energy consumption in both static [15-18] and dynamic context [19]. For more details on the PRT literature, the reader is referred to $[1,20]$. 
In the literature, a number of PRT simulations studies were conducted and developed to test PRT feasibility and performance. Xithalis developed a Java-based simulator for PRT called Hermes [21]. Xithalis with his simulator compared the synchronous and asynchronous control alternatives for PRT systems. He proved that for PRT systems the synchronous option is a better choice than asynchronous option control. Consequently, we suppose in this paper the use of synchronous mode of control for PRT system. Andreasson [22] developed a PRT simulation model called "PRTsim." A large urban PRT network composed of 69 stations and a fleet of 900 PRT vehicles was evaluated by Göran Tegnér et al. [23] using the Simula-based simulator. A PRT simulator developed by Schneider [24] using the Circulator-CAD was used to evaluate several PRT proposals. Other PRT simulators include TrakEdit and miPRT [3]. The miPRT simulator includes battery constraints. However, this simulator was implemented specifically for the case of Masdar city without giving much details about its implementation. For a more detailed review of PRT simulation programs, see [25].

The literature related to PRT has two main shortcomings when compared to the strategic needs of urban mobility using on-demand transportation tools.

First, most literature (except the miPRT simulator) related to the real time fleet management and dynamic routing of PRT pods does not consider battery issues. In fact, a limited battery capacity is considered as a complicated constraint for PRT system as vehicles would have only a limited distance to travel before returning to the charging location.

Also, benchmark models for mean waiting time of PRT system [7] are based on queuing models, Markov decision process, and static routing problems. However, static routing problem to benchmark mean waiting time of PRT systems does not consider several real-world constraints such as limited battery, distance, and time window constraints.

1.2. Objectives. The focus of this paper is on vehicles empty movement and passenger waiting times of PRT systems.

The demand responsive characteristic of PRT reflects its time sensitivity. This feature provides a great challenge to the PRT system in order to provide a fast and quick response to its customers' requests. The dynamically changing transportation service requests, such as continuously new transporting demands from different PRT stations, are very likely to result in increased transportation costs and longer waiting time for PRT users. Consequently, the decision-making problem concerns how to adjust the flow of empty available vehicles in the PRT system dynamically in order to cope with the real time transportation requests. This problem is a highly complex one due to several difficulties:

(1) Based on the PRT condition to respond and serve all requested transportation service from its customers, the question arises on how to reroute and move empty vehicles in order to serve all of them.

(2) As the response of the PRT system to transportation requests should be quick enough, one could ask about when and how quickly should the PRT central control system respond to them. Is it more beneficial for an empty vehicle to wait in its current station waiting for a possible passengers to come or will it be more favorable to the whole system that an empty vehicle should be sent as soon as a transportation request arises.

Traditional public transportation manager systems within a highly dynamic context as the PRT are not that flexible to deal with the different changes that could happen to the PRT environment. Consequently, a PRT manager might fail to cope with the above challenges and mentioned problems related to PRT. Therefore, it becomes of high interest to develop and formulate a robust DSS and a computer-based simulation with specific and adapted empty vehicles dispatching strategies which are capable of dealing with the dynamic characteristics of PRT.

The problem of dynamically managing empty vehicles within the PRT can be tackled as a dynamic routing and scheduling problem. Nevertheless, only few studies concerning empty vehicles management in PRT have been published $[7,8]$.

One should note that the integration of battery feature of vehicles, energy consumption, and passengers behavior in stations are among the different features that were not fully treated in the literature and represent specific aspects that our proposed DSS and simulation model bring in comparison to other PRT simulators that exist in the literature.

Finally, this paper develops a valid lower bound on mean passenger waiting times using a mathematical formulation. In fact and starting from a static context of PRT, we define a new deterministic routing problem taking into account different aspects of PRT such as distance, battery, and time windows constraints. Our problem is related to the vehicle routing problem (VRP), and it is NP-hard. Based on this deterministic routing problem, a mathematical formulation is proposed to provide valid lower bounds on mean passenger waiting times. Lower bounds on mean waiting time for PRT system are of a high interest in order to assess the performance of fleet management strategies in dynamic context. In fact, these lower bounds might give us insights about the performance of PRT systems and its operability in different urban contexts which might be independent of the algorithms used to manage it.

The focus of this paper is on efficiently managing the PRT system by paying special attention to passenger waiting times and the energy wasted by the movement of empty vehicles. In fact, the volume of passenger demand is often unbalanced across stations in a PRT system. Furthermore, the number of vehicles departing from a given station is not necessarily equal to the number of vehicles arriving at that station. Thus, many vehicles move while empty, which results in a significant amount of wasted energy. Therefore, it becomes important to know which PRT pods to move and where to move them with respect to the many constraints on the PRT system. This problem is called the empty vehicle redistribution (EVR) problem [26, 27]. This paper is concerned with the empty vehicle redistribution problem for a PRT system that uses vehicles with limited battery capacity in both its static and dynamic versions. More specifically and in the literature related to the real-world application of VRP, 
we could distinguish two main dimensions: evolution and quality of information. Evolution is related to the fact that the information related to the decision maker could cha nge during the execution of routes, whereas quality of the information is related to the uncertainty related to the available data for the decisions maker such as the arrival of new passengers requests. The dynamic problem is related to the fact that the PRT system receives new passengers requests as the time goes by. Static deterministic problem is related to the fact that the passengers demands are known beforehand and no changes happen during the execution of the routes. More details about the classification of real-world applications of VRP could be found in Pillac et al. [28]. This represents an important problem for PRT as the battery issue is often overlooked in the literature. In fact and in some PRT stations, PRT pods could not be dispatched to serve transportation requests originating from its current location only because it does not have enough electric energy to serve this specific transportation request and return to the depot. Also using vehicles with limited range would make part of a PRT fleet unavailable to serve transportation requests due to the charging operations. Consequently, this feature introduces a level of uncertainty within the system. This uncertainty makes the proposed problem and how to deal with it different from the actual solution approach adapted for vehicles without limited battery capacity.

Consequently, we start first by proposing a static deterministic routing problem in order to provide benchmarks on waiting times of passengers in PRT.

We attempt to know whatever the proposed mathematical formulation able to provide good solutions for our problem and to know the impact of the different parameters on the performance of our mathematical formulation.

We also study in this paper a dynamic EVR routing problem as we consider that more passengers arrive while the system is operating. The dynamic EVR problem is of considerable interest as it plays an important role in operational decisions for PRT systems. Accordingly, this paper develops an efficient PRT simulator which is able to simulate the different and rich aspects of a PRT system. We wanted to provide several practical details about developing our simulation model in order to enrich the existing literature of PRT simulators. Finally, the developing of a DSS taking into account the several dynamic and complex aspect of PRT is of a high interest in order to improve the decision-making process related to such a transportation system.

1.3. Contributions of This Paper. In this paper we do the following:

(1) Propose a new problem formulation in order to minimize the waiting time of passengers in the context of PRT in a static deterministic context. Studying this static problem offers the possibility of proposing valid lower bounds and benchmarks for evaluating dynamic strategies of routing PRT vehicles. To the best of our knowledge, the static PRT problem to minimize waiting time of passengers under battery and time window constraints was not studied in the literature. This work extends recent studies $[7,15]$ on PRT by defining, characterizing, and modeling this new related transportation problem and applying it to the PRT mode in urban areas.

(2) Design a discrete event simulation model for PRT. In the simulation model, we define a centralized option to control and manage the fleet of PRT vehicles. We also use different extensive techniques to model the behavior of the PRT system in real time based on the synchronous mode of control.

(3) Develop different proactive and reactive empty vehicle redistribution management strategies that satisfy passengers transportation demands while minimizing empty movements and wait times.

(4) Verify the performance of our model and our strategies through extensive computational tests and scenarios.

(5) Propose a DSS based on our developed simulator and developed strategies with emphasis on solving the dynamic EVR which captures several operational problems and features arising for PRT in the real context comprehensive dynamic.

The rest of this paper is organized as follows: Section 2 presents the PRT system and its related decisional problems. In Section 3, we describe the problem at hand as well as a valid mathematical formulation in order to provide valid benchmarks for mean waiting time of passengers based on a related static deterministic context. Section 4 follows to introduce the architecture, the proposed traffic management strategies used for PRT systems, and the core modules of the proposed DSS. Section 5 contains definitions of the concepts of a simulation and a discrete event simulation. We also describe our proposed simulation model in this section. A discussion of the computational results of different strategies that we employed is provided in Section 6. We offer our conclusions in Section 7.

\section{The Personal Rapid Transit}

The PRT system is an automated public transport mode that uses small, driverless electric vehicles (see Figure 1). PRT deploys its fleet of vehicles (also called pods) on a dedicated network of PRT guideways with offline stations that are located off the main line allowing vehicles to travel nonstop from origin to destination (see Figure 2). In this manner, PRT offers passengers on-demand nonstop service.

Users of PRT systems move from one location to another by going to their nearest PRT station and selecting their destination. The system assigns the user a vehicle that takes $\mathrm{him} /$ her directly to the destination. This is possible because of the specific topology that characterizes the PRT network. The guideways in the PRT network are arranged to have several divergent and convergent points, in addition to PRT stations located along the main guideways. This permits the PRT system to offer a direct, nonstop point-to-point service where vehicles bypass all unnecessary intermediate stations between a passenger's origin and destination. 


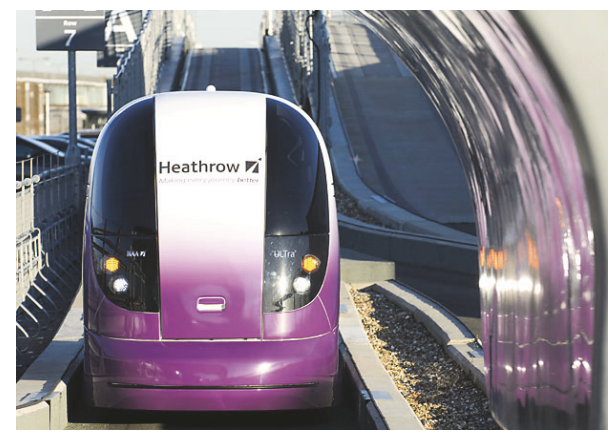

FIGURE 1: PRT vehicle (source: www.ultraglobalprt.com).

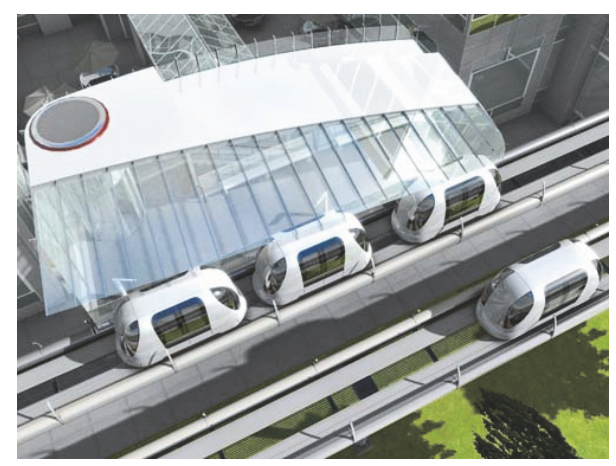

FIGURE 2: Offline station of PRT system (source: http://www.ultraprt .net/animationImages.htm).

PRT is suitable for small cities and can be used as a feeder for metropolitan train systems [29]. Three PRT systems are currently in operation: the "2getthere" PRT system in Masdar city in Abu Dhabi, United Arab Emirates (UAE), the Ultra Global PRT system at Heathrow Airport in London, United Kingdom (UK), and PRT SkyCube in Suncheon, South Korea.

2.1. Characterization of the PRT Decisional Problems. It is clear from the definition of the PRT system that it can be managed based on three different levels of decisions.

(i) Strategic level of decisions defines the decisions related to the PRT infrastructure like the guideways itinerary, station locations, and design under several budget and city regulation restrictions.

(ii) Tactic level of decisions defines the decisions that are taken periodically by the system manager such as the fleet size and the PRT schedule.

(iii) Operational level of decisions defines the set of decisions that are taken daily such as the optimization of the vehicles itinerary under several constraints such as battery, time windows, and capacity constraints.

Accordingly, this paper focuses on the operational level of decisions related to PRT. Therefore, we assume that all the strategic and tactical decisions related to PRT were already taken. This implies that a PRT network of a fixed guideways is already designed.
For a typical working day, the PRT needs to meet with the upcoming demand of passengers which is generally characterized as a Poisson process. As a consequence, two different modes of operation could be characterized (the Morgantown PRT system is a typical example of these two operation modes: http://assets.slate.wvu.edu/resources/1610/1406301617 .pdf).

(i) The demand mode of operation which is used when the demand of passengers is not predictable. Therefore, the system operates under uncertainty and runs based on the current demand of passengers.

(ii) The schedule mode of operation is used when the demand of passengers is predictable (as in rush hours). The PRT vehicles run according to a preset schedule based on a defined set of origin destinations moves.

The schedule mode is closely related to the static deterministic case related to PRT. It is useful to use and implement when the demand of passengers is prebooked. Therefore, vehicles would move based on a predefined set of origin destinations pairs. On the other hand, the demand mode is closely related to a dynamic routing problem which is useful to manage PRT vehicles in a dynamic setting.

Accordingly, we focus in what follows on the demand and schedule modes of operations related to PRT. In fact, we start by presenting the operational transportation problem related to PRT. Then, we treat the static deterministic version of this problem to minimize the waiting time of passengers. Finally, we treat the dynamic version of the transportation problem related to PRT by presenting a DSS and different reactive and proactive strategies for the real time fleet management of PRT pods.

Let us recall that Lees-Miller and Wilson [7] and Daszczuk et al. [8] proposed minimizing waiting time of passengers in a PRT context without considering battery constraints. Mrad and Hidri [15], Fatnassi et al. [17], and Mrad et al. [30] proposed minimizing energy consumption for a PRT system in a static deterministic context.

In this work we extend these PRT studies to our context. In fact as we deal with the schedule mode of operation, we propose minimizing waiting time of passengers under time window and battery constraints. To the best of our knowledge, this version of the problem was not proposed in the literature before. Also and in order to deal with the demand mode of operation, we propose a DSS, a discrete event simulator developed based on Anylogic and different proactive and reactive real time fleet management strategies while considering several constraints. To the best of our knowledge, a computerized DSS for PRT and a discrete event simulator taking into account battery and distance constraints were not proposed in the literature before for the PRT context. Consequently, this work extends the literature on the PRT to deal with the different PRT mode of operations while proposing both a static deterministic and a dynamic PRT transportation problems. 


\section{The Personal Rapid Transit Transportation Problem}

In this section, we first present the problem definition of our problem. Then, we present a short review of vehicle routing problems. We then present the proposed mathematical model in order to benchmark waiting time of passengers.

\subsection{Definition and Modeling}

3.1.1. The Business Context. First, let us focus on the specific business context of the PRT system. We should note that we aim to find the best tradeoff between its economic, societal, and ecological performance. We aim to study the efficiency of the PRT system in terms of waiting time for its passengers under various constraints and to study the behavior of a battery operated PRT system.

The PRT system, for a specific working day, offers a high quality on-demand transportation service. However due to a limited fleet size of PRT vehicles, the PRT system cannot ensure that a vehicle is present at each specific PRT station whenever a PRT user asks for it. This feature results in a large amount of empty vehicle movements and a large amount of waiting time for passengers which represent a huge loss in terms of economic and societal performance of such an intelligent transportation system.

For a given working day for PRT, transportation requests are received incrementally. Considering a PRT network in a specific urban area, a cost related to the energy consumption and the waiting time of passengers is associated with the decisions of dispatching vehicles to serve transportation requests. Given this cost, we introduce next the PRT problem definition.

3.1.2. Problem Definition. Our aim is to focus on the business context of PRT system by satisfying a set of transportation request. In this section, we focus exclusively on routing PRT vehicles that are running on battery power. The problem treated in this section is based on the following assumptions.

Let us consider a network of PRT guideways $N$. $N$ is composed of $M$ stations $S=s_{1}, s_{2}, s_{3}, \ldots, s_{m} \cdot|S|=M . N$ contains also one depot station $D$. $N$ ensures the connectivity constraints of any PRT network. This feature ensures for a PRT vehicle to be able to reach any stations from any other station. The transportation service in $N$ is ensured using a set of $K$ electric PRT vehicles which are initially located at $D$. The vehicles are battery powered. Each PRT vehicle has a limited electric battery capacity $B$ that makes it run for a limited duration in time. We assume that the PRT vehicles, whenever needed, go to the depot to recharge their batteries. We also assume that the consumed electric power from the depot to any station and going back to the depot does not exceed the battery capacity $B$.

Passengers arrive to the PRT stations following a Poisson process $\lambda$. The PRT system should respond favorably to all the transportation requests. A dispatching decision is needed for PRT system where there are empty waiting vehicles available at the PRT station and waiting passengers that need to be transported within the PRT network. Following the specific
PRT characteristics, a PRT vehicle could only serve one transportation request at a time. This transportation request consists of moving one passengers or a group of passengers up to six people from a specific departure station to a specific destination station in $N$. This feature makes the development of a specific sophisticated dispatching system for PRT an important task. The objective of our problem is to enhance the service quality of the system by minimizing its energy consumption and reducing the waiting time of passengers.

For our problem and as a first step, we try to minimize delays of service against the tie that the system is informed. Consequently, we suppose that the demand of passengers is known in advance. This is a very important objective for such an on-demand service, as the coming of passengers is supposed to be unknown in advance. In PRT, the case of perfect demand responding service also exists where each demand is already known in advance. Therefore, the scheduling and routing for each vehicles could be computed at the starting of the transportation service In this case, the obtained evaluation function could be used as an index to evaluate dynamic strategies. This is made possible as the static problem would present lower bounds for simulation strategies. In the literature, different studies were used to compare static and dynamic solution for vehicle routing problem [31] and PRT [32]. Generally, the static routing and scheduling problem could be presented as the following optimization problem.

The static PRT problem is characterized based on the business context defined above. Let us also define a list of known transportation requests $T$ of size $n$. Also, the matrix cost defines the least cost of moving between each pair of stations. The matrix SP defines the shortest time travel between each pair of stations. Each passenger demand is modeled by a request that contains information about the demand. To respond to passenger demand $i$, a PRT vehicle must go from departure station $\mathrm{DS}_{i}$ to destination station $\mathrm{AS}_{i}$. The service should start at least at the request time $\mathrm{RT}_{i}$ demanded by the passenger or later. The time of the start of service of a trip $i$ is denoted by $\mathrm{SS}_{i}$. Our aim is to design a set of least-cost vehicle routes capable of accommodating all the passenger requests under a set of constraints. The constraints include the time window and the battery capacity $B$ of the vehicles. The objective of our static problem is to minimize the waiting time of passengers. Consequently, our goal is to find a set of routes starting and ending at the depot covering all the trip demand exactly once with respect to the battery capacity of each vehicles in order to minimize the waiting time of passengers.

After presenting our static problem definition, we shall now present a formal theoretical graph representation.

\subsection{A First Basic Graph Representation for Our Problem. We} present, in this section, a first intuitive graph representation for our PRT problem.

This first representation is done by transforming the physical PRT network into a new network where a node represents either a departure station or an arrival station of a trip. This first graphical representation works by producing as many copies as needed of stations for each passenger request. 
To do this graphical transformation, let us introduce the following variables:

(i) A set DS $=\mathrm{ds}_{1}, \mathrm{ds}_{2}, \mathrm{ds}_{3}, \ldots, \mathrm{ds}_{n}$ is the set of departure stations of trips.

(ii) A set AS $=a s_{1}, a s_{2}, a s_{3}, \ldots, a s_{n}$ is the set of arrival stations for trips.

(iii) Each trip $i$ is represented by two nodes $\mathrm{ds}_{i}$ and as ${ }_{i}$ and a direct arc from $\mathrm{ds}_{i}$ to $\mathrm{as}_{i}$. The cost of this arc is equal to the total consumed energy following the shortest path from station $\mathrm{ds}_{i}$ to station $\mathrm{as}_{i}$. This set of edges is called $\mu$.

(iv) For each node $\mathrm{as}_{i} \mathrm{ds} s_{j}$, we add an arc from as $s_{i}$ to $\mathrm{ds} s_{j}$. The edge represents the empty move of vehicle after finishing trip $i$ to serve trip $j$. It has as a cost the cost of moving from $\mathrm{as}_{i}$ to $\mathrm{ds}_{j}$. This set of edges is called $\kappa$.

(v) For each node $\mathrm{ds}_{i}$, we add and edge from $D$ to $\mathrm{ds}_{i}$ representing the empty move from the depot to the departure station. This set of edges is called $\phi$. The cost of this arc is equal to the total consumed energy following the shortest path from the depot to $\mathrm{ds}_{i}$.

(vi) For each node $\mathrm{as}_{i}$, we add an edge $\mathrm{as}_{i}$ to $D$ representing the move from the arrival station to the depot. This set of edges is called $\iota$. The cost of this arc is equal to the total consumed energy following the shortest path from as $_{i}$ to the depot.

Therefore, we transformed our physical PRT network to a main graph $\Gamma$ composed by a set of node $\vartheta$ and edges $\varepsilon$ where

(i) $\vartheta=D S \cup A S \cup D$.

(ii) $\varepsilon=\mu \cup \kappa \cup \phi \cup \iota$.

We add for each node in DS and AS a specific time window indicating the time of service of trip $i$. We add also for each node in DS and AS a specific time window indicating the time of finish of service of trip $i$. We should note that in this graph there are a set of mandatory edges which are the edges representing the trips $\mu$.

3.3. Illustrative Example. Let us suppose that we have a PRT network as presented in the upper right corner in Figure 3. The edges represent the cost of moving between each pair of stations $A, B, C$, and where $D$ represents the depot. Let suppose that we have a set of trips as presented in the upper left corner in Figure 3. The resulting graph is shown in Figure 3. As an example trips 2 and 3 have the same departure station $B$. However in this graph representation, this station was replicated two times as we have two different trips.

3.4. VRP Related Literature. Starting from our business context and from our first intuitive problem modeling, we could note that our problem shares similarities with a famous subclass of VRP called VRP with pickup and delivery problems (VRPPD). In VRPPD, a set of vehicles must satisfy a set of transportation request. Each request is defined by a pickup point, a delivery point, and a specific demand to be transferred between the two locations. The transportation service could consist of transporting persons or goods. The objective involves generally the minimization of transportation and travel cost.

In the literature of VRPPD, there are three subclass problems:

(i) The many to many PDP where each location could be either a pickup point or a delivery point.

(ii) The one to many to one PDP where goods available at the depot location need to be transferred to the customer location. The goods present at the customer location need to be transferred to the depot.

(iii) The one to one PDP where each transportation request has a unique and specific pickup location and a specific delivery location.

Our PRT problem as it was already presented consists of moving a set of passengers from a specific departure location to a specific delivery location. Therefore, we could claim that our PRT problem shares similarities with the one to one PDP and a specific attention should be pair to this class of problems. The one to one pickup and delivery problem could be also divided into three subclasses [31]:

(i) When vehicle have the possibility of serving multiple location then this problem is a classical PDP.

(ii) When the transportation service consists of serving passengers and elderly people and the service is similar to a bus system, the problem is called a dial a ride problem (DARP).

(iii) When the vehicle could serve only one request at a time (as in our PRT case) then the problem is called the stacker crane problem (SCP).

The SCP takes its name from the practical problem of moving crane in ports, factories, and so forth. Until now the main application of the SCP was to manage truck fleet to move truck load between different locations that is why the SCP is also called the full load PDP. The objective of this problem is to minimize the empty moves.

Our PRT problem could be considered as an extension to the SCP as we add to the classical SCP different new constraints such as battery constraints and time window constraints; also we introduce a new objective as we aim to minimize the waiting time of passengers. In the next subsection and starting from these similarities with the SCP problem, we present a more enhanced and compact graph modeling of our static PRT problem.

3.5. A Second Enhanced Problem Modeling. The first and basic modeling of our PRT problem could be enhanced if we considered representing each trip as one node instead of two nodes and a mandatory edge. This will have as an impact to reduce considerably the number of nodes and edges and therefore reduce the size of the graph.

To solve our problem, we model it as a more compact theoretical graph problem. Let $G=\{V, E\}$ be a nondirected graph in which the vertices $V=\left\{v_{0}, v_{1}, v_{2}, \ldots, v_{n}\right\}$ correspond to the depot and the different passenger requests. 


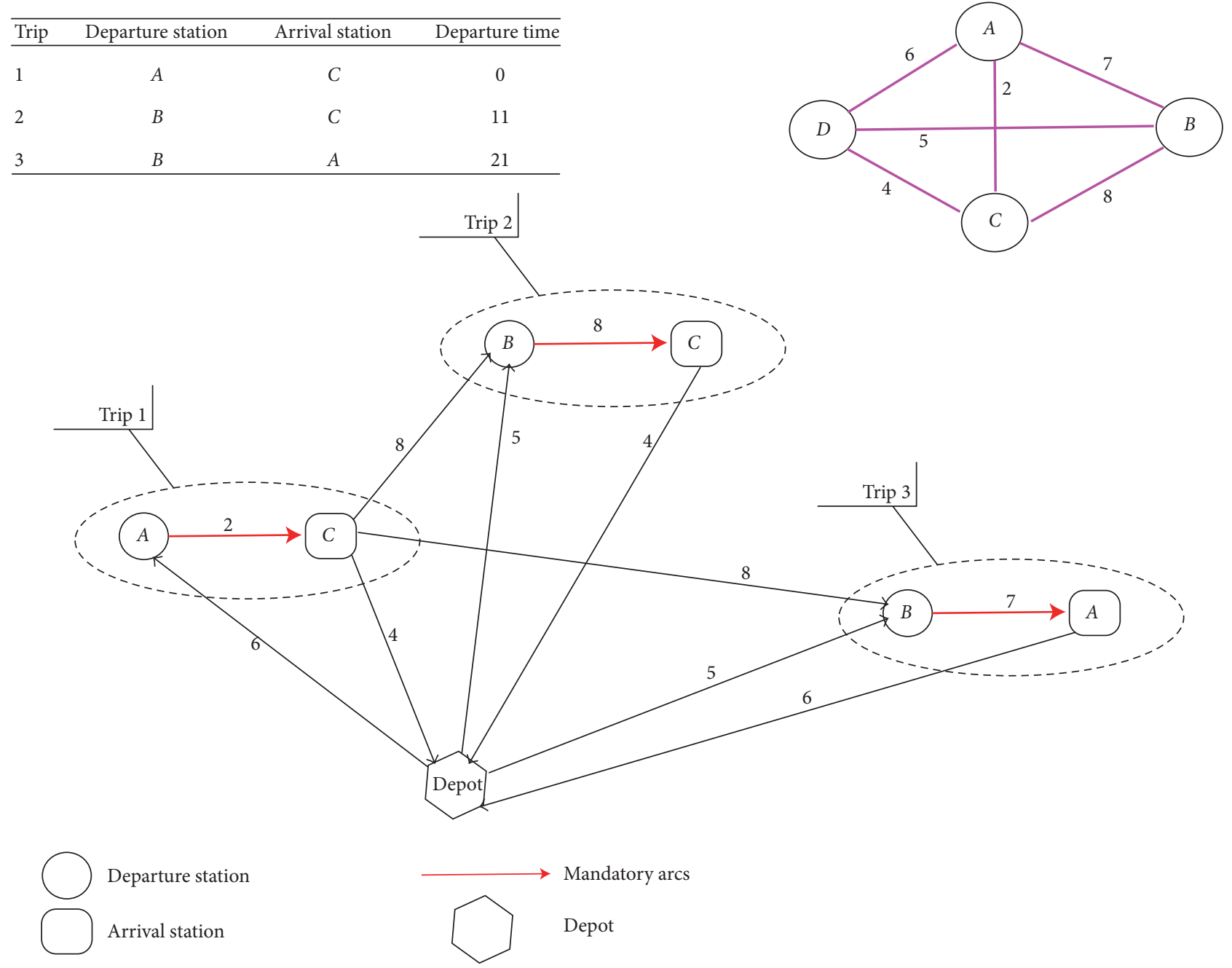

FIgURE 3: First illustrative example.

$V^{*}=V \backslash\left\{v_{0}\right\}$ represents the set of passenger requests, and the vertex $v_{0}$ represents the depot. The edges $E=(i, j) ; i, j \in V$ represent the set of links between different vertices. The edges $E^{*}=(i, j) ; i, j \in V^{*}$ represent the set of links between different vertices representing a trip. More specifically, the set of edges is defined as follows:

(i) For each pair of nodes $i, j \in V^{*}$, a link between them exists. This link has the associated cost of moving from the arrival station of trip $i$ to the departure station of trip $j$ in addition to the cost of moving from the departure to arrival station of trip $j$.

(ii) For all nodes $i \in V^{*}$, we also add a link $(0, i)$ whose cost is the cost of moving from the depot to the departure station of trip $i$ plus the cost of moving from the departure to arrival station of trip $i$.

(iii) Finally for all nodes $i \in V^{*}$, we add a link $(i, 0)$ whose cost is that of moving from the arrival station of trip $i$ to the depot.
From this graph representation of the problem, we can see that it shares similarities with the asymmetric distanceconstrained vehicle routing problem (ADCVRP). The ADCVRP is a variant of the VRP problem, in which the total distance traveled by each vehicle must be less than or equal to a maximum distance or time (in this case, the battery capacity). The objective of this problem is to minimize the travel costs. This problem has been proven to be NP-hard [33, 34]. Our problem is asymmetric because the distance between node $i$ and node $j$ is different to that from node $j$ to node $i$. Our problem extends the ADCVRP as we add different time windows constraints and we aim to minimize the waiting time of passengers rather than the traditional total travel distance which constitutes a new objective function for the ADCVRP. Our PRT problem represents also a new application of the ADCVRP.

3.6. Arc Flow Formulation. In this section, we present a based arc flow mixed integer programming formulation for solving our problem based on our second enhance problem modeling. It is based on existent works on ADCVRP $[33,34]$ and 
PRT [15] in the literature. Recall that we want to minimize the waiting time of passengers in a static deterministic context under time window, distance, and battery context. To introduce our valid mathematical formulation for our PRT problem, let us first introduce the following variables:

(i) $c_{i j}$ is the cost of going from node $i$ to node $j$ in the graph $G$.

(ii) $\delta^{+}(i)$ is the set of edges that have $i$ as a root.

(iii) $\delta^{-}(i)$ is the set of edges that have $i$ as a sink.

(iv)

$$
x_{i j}= \begin{cases}1 & \text { if node } j \text { is visited after node } i \\ 0 & \text { Otherwise. }\end{cases}
$$

(v) $z_{i j}$ is the least consumed energy used to travel from the depot to trip $j$ as $i$ is the predecessor of $j$.

Let us recall also that

(i) $\mathrm{SS}_{i}$ is the time of service of trip $i$.

(ii) $\mathrm{RT}_{i}$ is the demand time of the request of passenger(s) to perform the trip $i$.

(iii) $K$ represents the number of vehicles available initially at the depot.

(iv) SP is the matrix of shortest travel time between each pairs of stations.

(v) $\mathrm{DS}_{i}$ is the departure station of trip $i$.

(vi) $\mathrm{AS}_{i}$ is the arrival station of trip $i$.

Hence the minimum waiting time for passengers covering all the trips with respect to the battery time window and distance constraints is the optimal value of the following linear program:

$$
\begin{aligned}
& \text { PRT (1): Minimize } \sum_{(i) \in T} \mathrm{SS}_{i}-\mathrm{RT}_{i} \\
& \sum_{j \in \delta^{+}(i)} x_{i j}=1 \quad \forall i \in V^{*} \\
& \sum_{j \in \delta^{-}(i)} x_{j i}=1 \quad \forall i \in V^{*} \\
& \sum_{i=0} x_{i j} \leq K \\
& \sum_{i=0} x_{j i} \leq K \\
& \sum_{(i, j) \in E^{*}} z_{i j}-\sum_{(i, j) \in E^{\prime}} z_{j i}-\sum_{j \in V^{*}} c_{i j} x_{i j}=0 \quad \forall i \in V^{*} \\
& z_{i j} \leq\left(B-c_{j 0}\right) x_{i j} \quad \forall(i, j) \in E \\
& z_{i j} \geq\left(c_{i j}+c_{0 i}\right) x_{i j} \quad \forall i \neq\{0\}, \forall(i, j) \in E^{*} \\
& z_{0 i}=c_{0 i} x_{0 i} \quad \forall i \in E^{*} \\
& x_{i j}\left(\mathrm{SS}_{i}+\mathrm{SP}\left[\mathrm{DS}_{i}\right]\left[\mathrm{AS}_{i}\right]+\mathrm{SP}\left[\mathrm{AS}_{i}\right]\left[\mathrm{DS}_{j}\right]-\mathrm{SS}_{j}\right) \leq 0 \quad \forall i, j \in V^{*} \\
& \mathrm{RT}_{i} \sum_{(j) \in \delta^{+}} x_{i j} \leq \mathrm{SS}_{i} \quad \forall i \in V^{*} \\
& 0 \leq \mathrm{SS}_{i} \quad \forall i \in V^{*} \\
& x_{0 i} * \mathrm{SP}[D]\left[\mathrm{DS}_{i}\right] \leq \mathrm{SS}_{i} \quad \forall i \in V^{*} \text {. }
\end{aligned}
$$

Equation (2) aims to minimize the differences between the time of service and the time of receiving the requests. This is equal to the waiting time of passengers of the different trips. Constraints (3) and (4) are the assignment constraints.
It ensures that each node representing a trip is visited only once. Constraints (5) and (6) ensure that exactly $K$ vehicles are used. Those constraints make sure that no more than $K$ vehicles leave and return to the depot. 
Constraints (7) represent the connectivity and subtour elimination constraints. They guarantee that the obtained final solution does not contain subroads, while also balancing inflow and outflow at each trip node.

Therefore, constraints (7) ensure that the electric energy used to travel from node $i$ to any node $j$ in a road should be equal to the difference between the energy consumed to travel from the depot to node $j$ and the distance traveled from the depot to node $i[34,35]$. These typical flow constraints were introduced in the literature for the problem of ADCVRP in order to eliminate illegal roads.

Constraints (8) are distance constraints. Constraints (8) make sure that the energy used to reach trip $j$ from the depot and the direct link from the depot to node $j$ are less than or equal to the battery capacity of PRT pods.

Constraints (9) ensure that the energy used to travel from the depot to trip $j$ is greater or equal to the energy used from the depot to trip $i$ in addition to the energy used from trip $i$ to trip $j$.

Constraints (10) present the initial values of variables $z_{D i}$. Constraints (11) are the schedule constraints. They guarantee the schedule feasibility for our PRT system. Those constraints take into account time consideration. Therefore, if the edge $x_{i j}$ is selected, the time of start of service of trip $j$ is equal to the time of start of service of trip $i$ in addition to the time needed to travel from trip $i$ to trip $j$.

Constraints (12) ensure that the time of service of trip $i$ is greater or equal to the time of receival of trip $i$.

Constraints (13) are time windows constraints and ensure that the time of service of any trip is later than the earliest time window.

Constraints (14) ensure that if a trip is served directly from the depot, its time of service is at least equal to the time needed to travel from the depot to its departure station.

A linearization of constraints (11) could be done as follows:

$$
\begin{aligned}
& \left(\mathrm{SS}_{i}+\mathrm{SP}\left[\mathrm{DS}_{i}\right]\left[\mathrm{AS}_{i}\right]+\mathrm{SP}\left[\mathrm{AS}_{i}\right]\left[\mathrm{DS}_{j}\right]-\mathrm{SS}_{j}\right) \\
& \quad \leq\left(1-x_{i j}\right) \text { BIGM } \forall i, j \in T,
\end{aligned}
$$

where BIGM is a large number. This could be done as we have an integer decisions variables (the set of variables $x_{i j}$ ).

\subsection{Performance of the Mixed Integer Formulation}

3.7.1. Plan of Experiments. To test our mixed integer mathematical formulation proposed to our specific PRT problem, several instances were generated based on the following dimensions:

(1) Trip list size

(2) Battery capacity

(3) Fleet size

(4) Network characteristics

The list of trips size was generated based on the instance generator from the literature [15]. The set of generated trips assumptions is presented in Table 1 Let us recall that a trip is

\begin{tabular}{|c|c|}
\hline PRT feature & Assumption \\
\hline Number of stations & 15 based on the Corby network \\
\hline Departure stations & Generated randomly between 1 and 15 \\
\hline Arrival stations & $\begin{array}{l}\text { Generated randomly between } 1 \text { and } 15 \text { while } \\
\text { ensuring that departure and arrival station } \\
\text { for each trip are different }\end{array}$ \\
\hline Departure time & Generated randomly between 1 and $3600 \mathrm{sec}$ \\
\hline Arrival time & $\begin{array}{l}\text { The sum of depart time and the duration of } \\
\text { the trip }\end{array}$ \\
\hline
\end{tabular}

TABLE 1: Generated trips assumptions.

defined by departure station, arrival station, departure time, and arrival time.

The size of trips ranges from 10 to 50 in a multiple of 10 . For each problem size and each network, we generated 3 different instances. The network was adapted from the PRT Corby network (see Figure 4) which is a realistic test case study developed by Teychenne and Bly [36].

It was used to test PRT solution in Northampton in the United Kingdom (UK). Relative data of this network are available in the ATS/CityMobil tool (see for instance http:// www.ultraglobalprt.com/about-us/library/ultra-simulator/) which was developed to test different dynamic strategies for PRT. This network proposes a topology with four different depots. As in our problem we suppose to have only one depot; we generated four different networks taken into consideration, one different depot at each time. Thus, we get four different networks.

As for the size of the fleet, we used three different sizes of the fleet $\chi$ based on the problem size (nb-trip) which were

(i) $\chi=$ nb-trip.

(ii) $\chi=$ nb-trip $/ 2$.

This will ensure having a possible realistic value for the size of the fleet.

Finally for the battery capacity, we generated three different battery capacities: 40,45 , and 50 minutes $[15,30]$ (in the paper of Mueller and Sgouridis [3], it is noted that battery capacity is $20 \mathrm{kWh}$, minimum recharge level is $50 \%$, and average vehicle power consumption is $250 \mathrm{Wh} / \mathrm{km}$; as we consider having a set of homogenous vehicles these assumptions are considered to be realistic, considering other values for the battery capacity do not diminish the applicability of the proposed model). The combination of these four dimensions yields 360 different testing instances. Each instances is denoted by a quadruplet $\psi, \chi, \varphi, \zeta . \psi \in\{1,2,3,4\}$ which represents the network characteristics. $\varphi \in\{10,20,30,40,50\}$ which represents the trip list size. $\chi \in\{$ nb-trip/2,nb-trip $\}$ which represents the fleet size. $\zeta \in\{40,45,50\}$ which represents the battery capacity.

3.7.2. Performance of Our Mixed Integer Formulation. Tests were coded in $\mathrm{C}++$ in visual studio 2008 on a computer with a 3.2 GHZ CPU and 8 GB of RAM. In addition, IBM-Cplex 12.2 was used to solve the different integer linear programs. 




Figure 4: The Corby network.

Results of our mathematical formulation are presented in Figures 5, 6, and 7. Figures 5(a) and 5(b) present the mean waiting time for each class of size of trips. We should note that we put a time limit of 600 seconds for IBM-Cplex which means that if the program exceeds 600 seconds without finding an optimal value, it returns the best feasible value founded so far.

Based on our results, we could note the good performance of the PRT system in terms of mean waiting time of passengers. In fact and based on these instances, we found an average waiting time for passengers of 59 seconds. This is true especially for instances related to the first network as we found an average waiting time of 34 seconds (see Figure 7). The relative average mean waiting time gives a good indication about the possible mean waiting time for real case PRT system that uses vehicles with batteries.

Obviously and from the obtained results, the depot location has its impact on the whole performance of the system. In fact, a change from the first network to the second network results in an increase of $72 \%$ in the mean waiting time. 


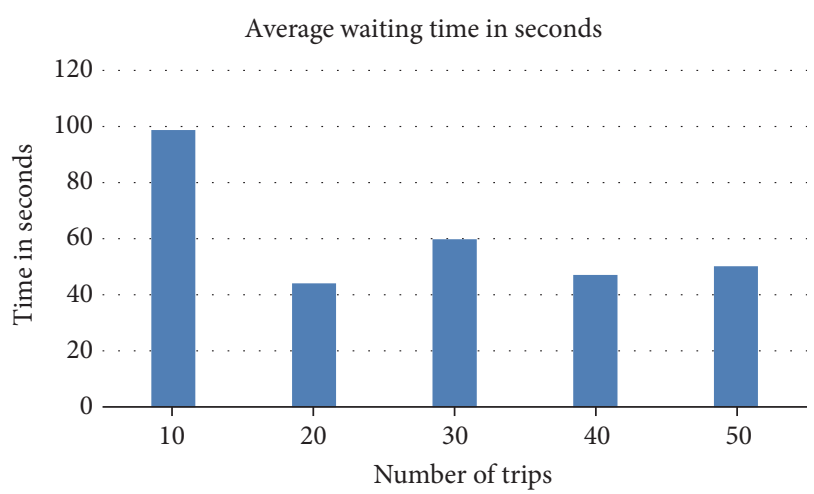

(a) Evaluation of the average waiting time in seconds for each size of list trips

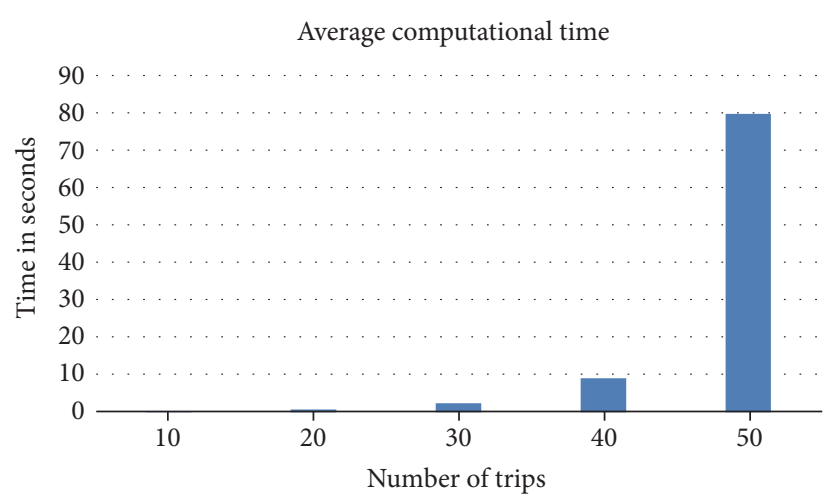

(b) Evaluation of the mean Computational time in seconds for each size of list trips

FIGURE 5: Pictures of grouped analysis for each size of list trips.

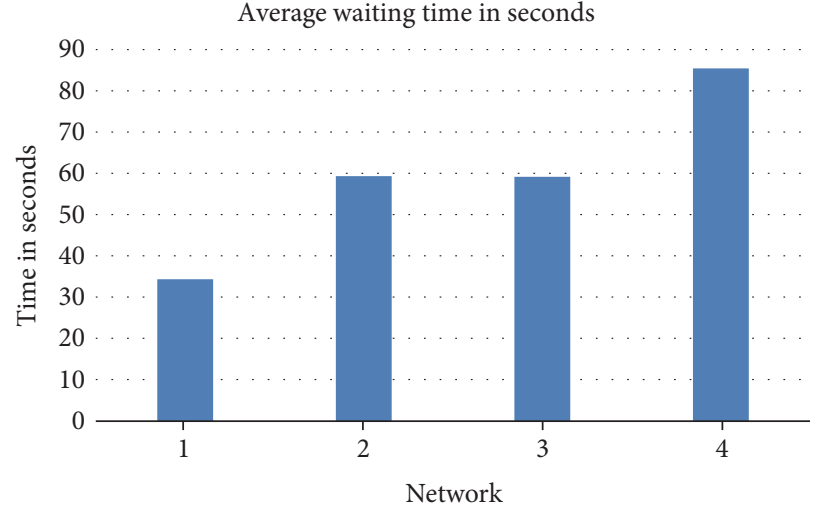

(a) Evaluation of the average waiting time in seconds for each network

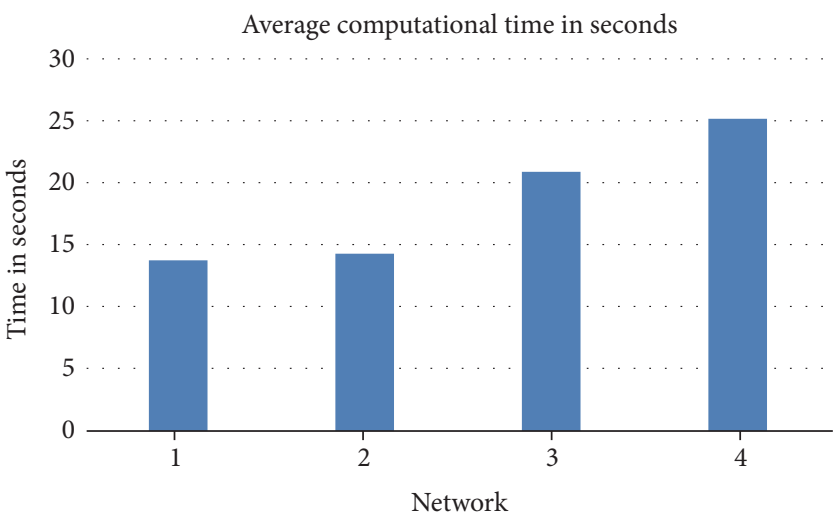

(b) Evaluation of the mean Computational time in seconds for each network

FIGURE 6: Pictures of grouped analysis for each Network.

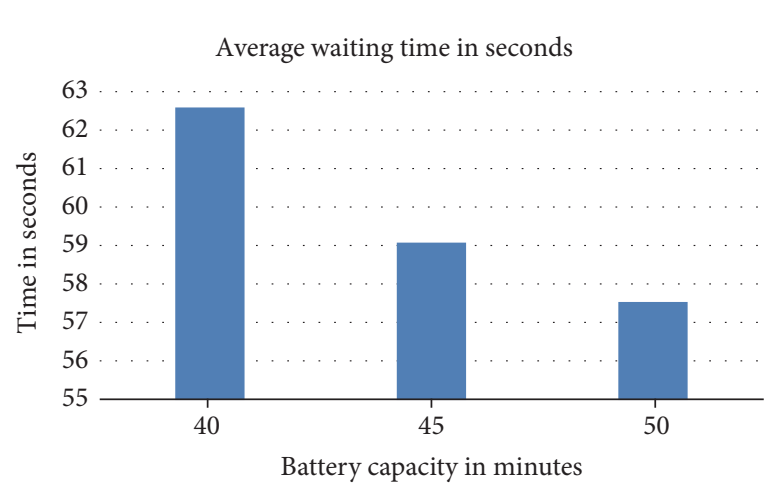

(a) Evaluation of the average waiting time in seconds for each battery capacity

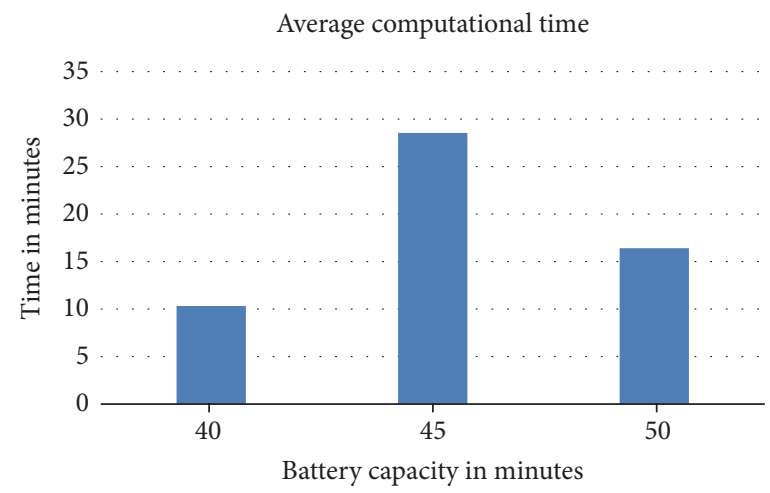

(b) Evaluation of the mean computational time in seconds for each battery capacity

FIGURE 7: Pictures of grouped analysis for each battery capacity.

Based on Figure 6, a change from the fourth PRT network to the first PRT network results in a decrease of $147 \%$ (this statistic was computed using the gap metric based on the following formulas: GAP $=\left(\left(\right.\right.$ Max $_{\text {waiting time }}-$ $\left.\left.\mathrm{Min}_{\text {Waiting Time }} / \mathrm{Min}_{\text {Waiting Time }}\right) \times 100\right)$ in the mean waiting time. Figure 6 suggests that when the depot is located in a central zone close to different stations performance of the system increase as it would be able to provide much smaller waiting time for its passengers. In fact, we could note that the average travel time between the first depot and the different stations 


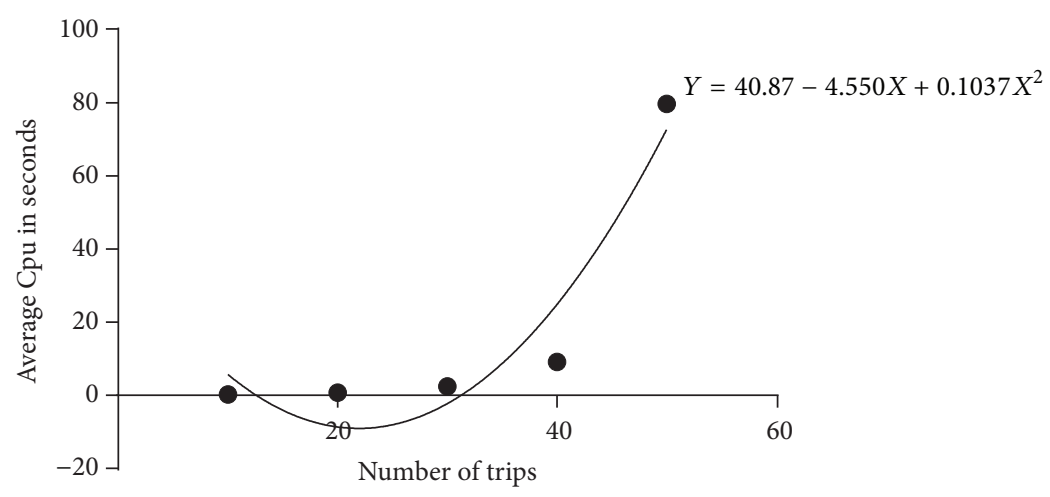

FIGURE 8: Results of the nonlinear regression.

is 299 seconds while it is 495 seconds for the fourth depot which corresponds to the worst performance in terms of mean waiting time of passengers. This analysis indicates that in terms of level of service, it is preferable to locate the depot in a central area close to the different PRT stations rather than in suburban areas. This fact also helps to reduce total travel time for vehicles. This feature combined with the fact that the guideways are dedicated to the PRT vehicles, helps to reduce congestion in cities centers. Locating PRT depot in suburban areas translates into larger travel distance, wasted energy, and empty movement for PRT pods to the city centers. However, lower depot costs come with the implementation of PRT depots in suburbs. This is mainly a consequence of the fact that land is cheaper in peripheral zones than in cities centers. Nevertheless, locating PRT depots into city centers yields overall several environmental and societal benefits. These results shows the importance of the tactical and strategic decisions related to the network and station design on the operational performance of the system. This also confirms results from the literature that show the importance of the depot locations on the operational performance of transportation systems [37].

Figure 7 provides an example of the impact of the battery capacity on the mean waiting time. Increasing the battery autonomy by 5 minutes from 40 to 45 minutes helps to decrease the mean waiting time by $5 \%$. Also, a decrease of $8 \%$ was observed when having a battery autonomy of 50 minutes over instances with battery autonomy of 40 minutes. Therefore, we observe a positive correlation between the waiting time and the battery capacity. This is mainly due to the fact that the longer the battery lasts, the least the vehicles would be unavailable due to charging issues.

Figure 5 presents the impact of the number of trips on the mean waiting time of passengers. We should note that in our case the size of fleet is related to the number of trips to serve. Consequently, we found smaller waiting time in large instances size than smaller instance size as larger fleet results in small waiting time for passengers. These results confirm the ones from the literature for vehicle routing problems [38].

Next, we wanted to analyze the relation that could exist between the size of test instances and the computational time
TABLE 2: Results of the Spearman correlation test.

\begin{tabular}{lc}
\hline Statistic & Value \\
\hline$r$ & 1 \\
$P$ (two-tailed) & 0.0167 \\
Significant? (alpha $=0.05)$ & Yes \\
\hline
\end{tabular}

TABLE 3: Results of the runs test.

\begin{tabular}{lc}
\hline Statistic & Value \\
\hline Points above curve & 3 \\
Points below curve & 2 \\
Number of runs & 4 \\
$P$ value (runs test) & 0.9 \\
Deviation from model & Not significant \\
\hline
\end{tabular}

of our proposed mathematical formulation. In fact, we wanted to test the influence of the number of trips on the performance of the proposed mathematical formulation.

For this purpose, a correlation test was conducted between the number of trips and the average computational time of the mathematical formulation. A correlation test is generally used to observe the statistical relationships between two or more data values or variables. As the observed data do not follow a normality distribution, we used the Spearman correlation test. Results of this test (see Table 2) show that there is a strong correlation between these two variables which proves the statistical relation between the number of trips and the average computational time.

Next, we wanted to drive an equation that predicts the computational time of the mathematical formulation as a function of number of trips. To that end, we used the nonlinear regression model (second-order polynomial (quadratic equation)) (Figure 8). Let us recall that a regression model is one where data values are modeled by a function of different parameters and independent variables. Results of this regression analysis are provided in Figure 8. As a postanalysis of this regression model, we used the Runs test. Results of this Runs test are shown in Table 3 and prove that our data fits the proposed regression model. 
(1) Obtain routing and PRT information data.

(2) Build the cost matrix relative to the PRT routing information.

(3) Transfer the obtained data to the simulation component of the DSS.

(4) Simulate the system based on a specific chosen strategy and the PRT data.

(5) Display the obtained results relative to the PRT simulated system.

Algorithm 1: PRT-DSS.

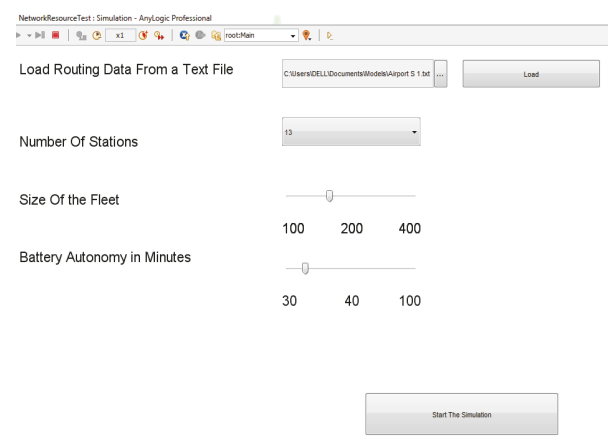

FIgURE 9: Data inputs of the DSS.

Finally, we should note that our mathematical formulation provides satisfactory results for our problem.

\section{A Decision Support System for the PRT}

In this section, we present the DSS proposed for PRT as well as the different implemented management strategies.

\subsection{A Full Integration of Management Strategies within the} Decision Support System. An implementation of a DSS for PRT aims at providing a sophisticated and flexible tool in order to choose the best dispatching strategy of PRT vehicles. Algorithm 1 shows the basic structure of our DSS.

Within our DSS, one question emerges on how to integrate the implemented dispatching strategies within our DSS. In the literature, several approaches exist to tackle the integration issue of optimization system within a generic DSS, namely, the loose, the full, and the tight integration. More details about this issue could be found in [39]. As it can be noted from Algorithm 1, our strategies are implemented based on the full integration method which embeds the optimization and dispatching module completely within the DSS. Consequently, the DSS will take full advantage of the implemented strategies in terms of speed of execution, analysis of the results, and so on.

Our DSS was implemented as a central intelligent system which is responsible for fully assisting the PRT manager to dispatch, schedule, and reschedule different operations related to PRT.

Our DSS starts by obtaining the input data such as the matrix cost and the number of vehicles and the battery capacity. Figure 9 presents an illustration on the input screen of our DSS. These data represent the input of the simulation step and for the different strategies implemented within our DSS. Such dispatching strategies used in the simulation step could be based on either basic or more sophisticated rules.

Outputs of our DSS will be shown to the PRT manager in different formats in such a way that PRT issues and problems could be dealt with and dispatching strategies could be analyzed properly.

4.2. Management Strategies. In the context of our research objectives, management strategies are simple rules to assign vehicles to a set of trip requests.

To deal with relevant problems, simple rules are applied each time there is a change in the state of the system. A change occurs when a new passenger request is received or if a PRT pod becomes empty.

In this section, we define four simple, classical strategies for minimizing empty moves and passenger wait times.

(i) First-come, first-served (FCFS): when a vehicle is available for a trip, it serves the passenger who has been waiting the longest.

(ii) Nearest neighbor $(\mathrm{NN})$ : passengers demands are served in order of their arrival at a station. Each vehicle serves the passenger request from the station closest to its own location.

(iii) Wait, then go (WG): after serving a passenger request, a vehicle will wait in its station for a passenger for a given time. If there is no trip request, the vehicle will serve its nearest passenger request based on the $\mathrm{NN}$ strategy. Consequently, the WG offers the combination of the principles of NN strategy with more focus on the waiting time of passengers. In fact, making the vehicles wait for potential passengers to arrive at a particular station helps the system to reduce the waiting time of passengers by providing a waiting vehicles in each station.

(iv) Fixed number of vehicles in station (FNVS): this strategy maintains a fixed number of idle vehicles at each station. As soon as a station has a deficit of idle vehicles, the dispatch system will send to the station the nearest idle vehicle from a station that has a surplus of empty waiting vehicles. This idea of maintaining a target number of idle vehicles at each station was used by Anderson in [40]. We will apply this basic rule when the system receives new passenger requests or when new vehicles at a station become empty. Vehicles would be dispatched to passengers based on the FCFS principles. This strategy also offers the 


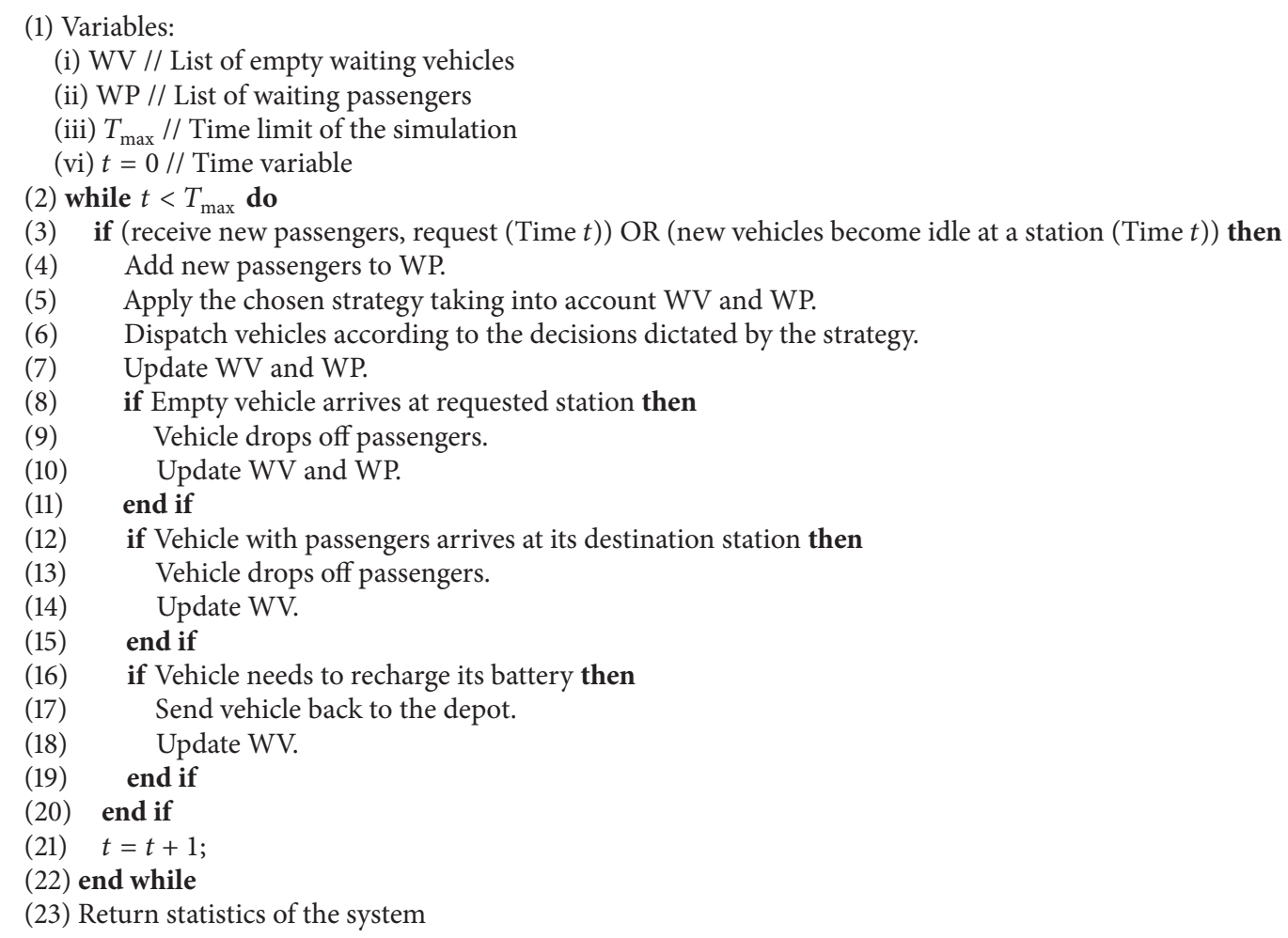

Algorithm 2: Simulation of the PRT System.

combination of the FCFS strategy (which is a reactive strategy) while offering a fixed number of vehicles in each station (which is based on proactive principles).

Finally, we provide an algorithm that describes the general steps and rules to simulate and manage our proposed PRT system (see Algorithm 2).

\section{Simulation}

Generally, real-world systems are typically too complex for simulation models to calculate their exact characteristics. Therefore, complex systems require numerical, computerbased simulations [41] to mimic the behavior of the real system. This allows the assessment, observation, and analysis of the system over time. Therefore, simulations play an important role in real-world systems as they aid the design process. Simulation is highly appealing because it makes it possible to mimic real-world systems [41] without the need to implement or build the actual system. Nowadays, a multitude of simulation software exists, making model building relatively easy. We should note that these simulation packages contain distinctive tools for analyzing simulation output. Finally, the evolution in hardware performance and software development enables simulation experts to conduct tasks quickly.

In this paper, we propose a simulation study of the PRT system. We do so by implementing a simulation model. As we are interested specifically in the management of the movement of empty vehicles in PRT, we use a discrete event simulation approach in our PRT simulation modeling. To implement a simulation model for a specific system, there is not only one solution available. Two models of the same system created by two different experts are highly unlikely to be identical. In fact and for the same system, one simulation model could be more bulky but sufficient while other simulation model can be more elegant but tricky. We believe that implementing a rather simple and straightforward simulation model for PRT makes it more efficient and easy to reproduce than other more complicated ones.

In the next section, we present our proposed simulation software. We will detail the main features of our simulation model, which has been developed to test our strategies, after which we will present the simulation results.

5.1. Simulation Software. Several types of simulation software are capable of generating high-quality simulation models. We examined numerous simulation software tools for thus study, such as prt-sim (source: https://code.google.com/p/prt-sim/), Hermes (source: http://students.ceid.upatras.gr/ xithalis/simulation_en.html), and so on. We chose the Anylogic simulation software to implement our simulation model because it is the only tool, to the best of our knowledge, that offers sufficient flexibility to use system dynamics, discrete events, and agent-based simulation methods within a simulation model. Thus, by using Anylogic, we can implement a model using discrete event simulation and extend it using 


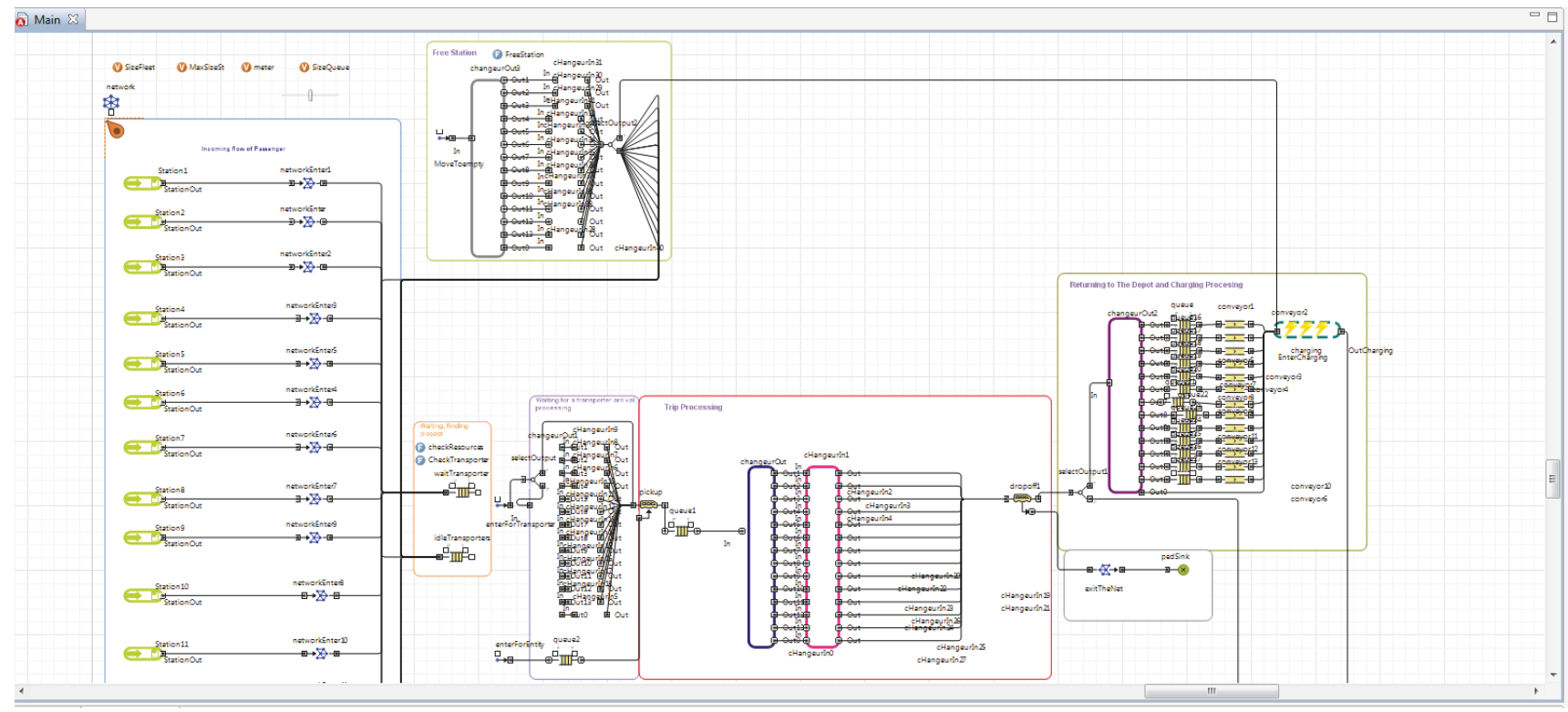

FIGURE 10: Simulation model.

agent-based methods. More precisely, Anylogic is a Javabased simulation platform that allows complex systems to be modeled through hierarchical and modular simulation models. Anylogic also provides a visual object environment and graphical interface to facilitate model building. It is a robust simulation tool that can be used to model economic market systems, social systems (behavior of customers), physical systems (manufacturing systems), and even logistics and transportation systems. The interface of Anylogic provides a visual language that includes Unified Modeling Language (UML) state charts, action charts, rich statistical tools, and an enterprise library that supports discrete event simulation. The enterprise library contains basic library objects, such as source, sink delay, queue, batch/unbatch, pickup/dropoff, thus making it possible to create several kinds of discrete event models. Even complex objects can be created through the library by combining multiple basic objects, which can be parameterized and assigned specific attributes. Another major advantage of Anylogic is its ability to create 2D or 3D animation for models. Indeed, each object has a default animation associated with it as a parameter. We can define, for example, an animation path for a certain object during a simulation run.

In the literature, several simulation studies were implemented using the Anylogic simulation software. One could note, for example, [42-46].

5.2. Simulation Model. The simulation model (Figure 10) is programmed as a discrete event simulation model based on and extended from [11, 47]. We develop two classes: the vehicle class that inherits from the Anylogic "entity" class and the passenger class that inherits from the Anylogic "pedestrian" class. We also define four different types of active objects:

(i) We associate an active object with each station to simulate the movement of the vehicle towards that station. (ii) To simulate the arrival of passengers in each station, we develop a station active object (see Figure 11).

(iii) We use an active object to represent the batterycharging operation in the depot.

(iv) The system also includes a "main" active object that contains the model itself.

5.3. The Network. The network is modeled using Anylogic conveyor objects. Conveyor objects have characteristics, like speed and length, that in our model represent a path from one station to another. The use of conveyor objects is suitable for PRT systems. Let us recall that the PRT vehicles use a set of exclusive guideways which separate the PRT traffic from any other urban traffic. As the conveyor object models the movements of an entities without considering other external factors, it makes the Anylogic conveyor suitable to model PRT guideways.

As soon as a vehicle begins to service a passenger, it is launched on the particular conveyor representing the path that it should take. To assign a vehicle to its relative conveyor, we use an active object for each station that represents all possible routes for the vehicle. The active object uses different decision points represented by the "SelectOutput" Anylogic object, whereby a vehicle can determine the precise conveyor to use according to its destination station. The distance matrix is a static member of the vehicle entity class and represents the cost of the shortest path between each pair of stations in the network. As this matrix is static, it can be accessed by any vehicle. Therefore, each vehicle can determine the cost of its movement. The 13 active objects that represent the movement of vehicles from stations are regrouped into a single active object that represents the whole track in the PRT network.

A SelectOutput object is placed before the active object representing the network so that the vehicle knows which 


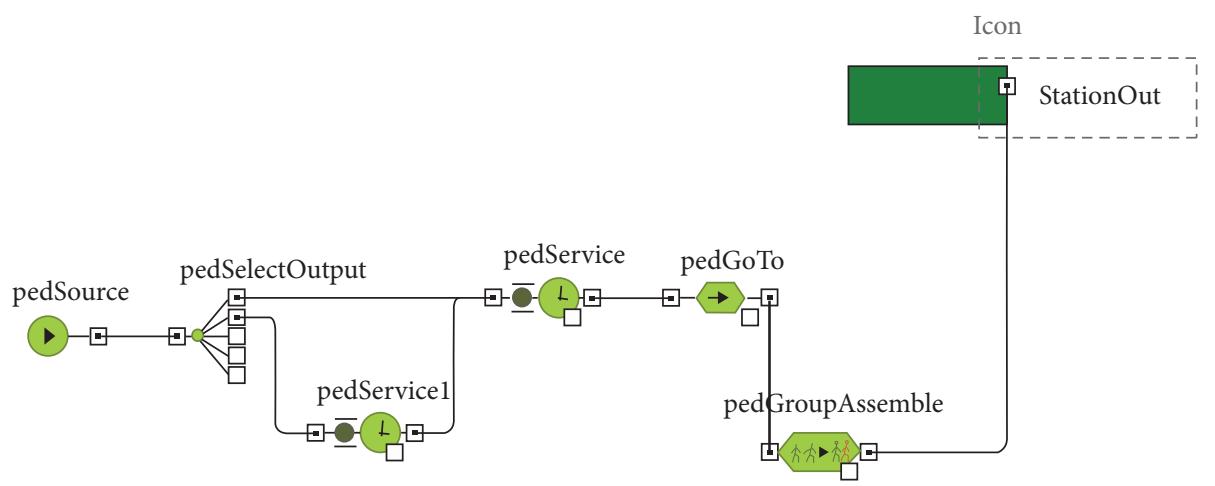

FIGURE 11: Station active object.

active object needs to be activated to initiate the simulation model.

With regard to the animation of the model, the network is drawn directly on the main class. The direct links between each pair of stations are traced separately and passed as an attribute to its relative conveyor object. Passengers waiting for a vehicle are modeled as a colorful point and are positioned directly at the appropriate station. Empty vehicles are shown in white and traveling passengers are shown in red.

The central station in the network is used as the depot, which is twice the size of the passenger stations.

5.4. The PRT Stations. To model the PRT stations, we focus on the passengers and the quality of service. Passengers are objects of the passenger class that implement the Anylogic pedestrian class. The passenger class has different attributes for any simple user of PRT, like their entry time, destination station, and so on. We use the pedestrian objects in Anylogic to model passenger behavior within a PRT station.

The PRT stations are modeled using a PedSource library block, two PedService objects, and a pedGroupAssembly object. The latter pedestrian Anylogic object is used to group passengers that arrive as a group to take a PRT ride.

We use two statistical objects to analyze the time spent by passengers in the station while requesting a service. The animation of passengers in a PRT station represents the area of a station and can also express the queue of waiting passengers asking for service. Each passenger in the PRT station animation is modeled as a graphical point.

5.5. Vehicles Entering a Station. PRT vehicles enter a station to drop off or to pick up passengers. Before entering a station in our model, a vehicle checks its destination and proceeds directly to the relevant station. Once the vehicle is in a PRT station, it leaves the PRT network as well as its animation and is transferred to the relevant station in the model. Two operations can be performed by a vehicle within a PRT station: an embarking operation, whereby a passenger enters a vehicle, and a disembarking operation, whereby passengers leave the vehicle in a station.

5.5.1. Embarking Passengers. For passengers to get into a PRT vehicle, our model uses an enterprise library Anylogic pickup object. When passengers have boarded, the passenger ID in the vehicle class changes to reflect the IDs of the passengers on board. Therefore, passenger attributes, such as wait time and destination, are transferred to the vehicle. The embarkation time is assumed to follow a uniform distribution between $30 \mathrm{~s}$ and two minutes. When the embarking operation is complete, the vehicle changes its status and its animation color changes to red. The departure time is recorded and added to its relative static objects.

5.5.2. Disembarking Passengers. Disembarkation is handled using the Anylogic dropoff object. As in the embarking operation, the time needed for disembarking is uniformly distributed between $30 \mathrm{~s}$ and two minutes. As the disembarking operation is executed, the passenger ID attribute in the vehicle is set to empty. Trip statistics, like the time needed, the number of passengers transported, and so forth, are recorded. Following the disembarking operation, the passenger objects exit the system through an exit network object and a pedSink object. Following the disembarking operation, the vehicle checks whether it needs to proceed to the depot to recharge its battery. This is verified by checking whether the current level of electricity in the battery is below a critical level. If so, the vehicle proceeds directly to the network and the depot is set as its destination station. Otherwise, the vehicle will be added to the central queue object that centralizes all idle vehicles.

5.6. Rerouting of Moving Empty Vehicles. Rerouting of empty PRT vehicles is handled using a specific implemented activeObject within the proposed simulation model. In fact and within the simulation, empty vehicles could be needed to be rerouted in order to take waiting passengers or in order to free a PRT station berth. To do so and based on the chosen strategy, decisions could be taken in order to move an empty vehicle from its current location to a destination location. To do so, the vehicles would be injected in an Anylogic "NetworkEntry" object. Also, the destination attribute of the PRT vehicle would be updated. Next and based on the destination of the vehicle, the implemented Anylogic dispatching would choose the related conveyor in order to send the vehicle to its destination. When it arrives to its destination, the status of the vehicle would be updated again in order to set it to free. Finally, and based on the situation of the current PRT station, 


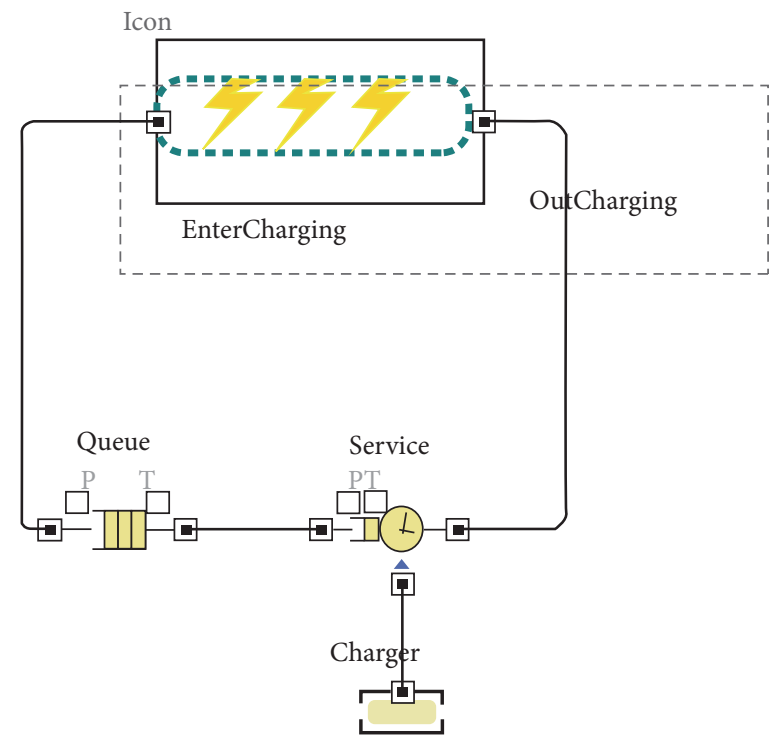

FIGURE 12: Charging active object.

a vehicle could be dispatched to move passengers to their destination.

5.7. Charging Operation. Charging is one of the most important features in PRT systems using battery-powered vehicles. Vehicles are sent to the depot to recharge if their energy levels are below a critical level. The charging facilities in the depot are modeled as a server object with a relative queue (see Figure 12). Vehicles are served in the depot according to a first-come first-served rule. We should note that the charging operation occurs using a ResourcePool Anylogic object. This defines the number of available charging slots in the system. The time needed for charging depends on the level of remaining charges in the battery. Once a vehicle starts its charging operation, it will not be released back to the network until the charging operation has finished.

\section{Simulation Results}

To verify the results and the performance of our four proposed management strategies, we designed tests based on the model described in the preceding sections. We compared the results of these tests to determine the best management strategy and show that our proposed strategies are effective.

6.1. Plan of Experiments. We assumed that all vehicles are initially located at the depot. We also assumed that the vehicles follow the shortest path from departure station to the destination station. Our simulation covers a period of three hours and was tested on a Windows 7 PC with an Intel i5 $3.2 \mathrm{GHz}$ processor and $8 \mathrm{~GB}$ of random-access memory (RAM).

To test the four management strategies to manage PRT, we used several test cases based on three different dimensions:

(i) Fleet size. (ii) Demand rate.

(iii) PRT network.

The PRT network was generated randomly but was based on realistic value ranges obtained from $[3,15,30]$.

More specifically, we used our model of 13 stations and one depot to generate the incidence matrix of the PRT network. We first generated randomly between [1,15 min] the durations of the movements between each pair of stations. Following this, we used the Floyd-Warshall algorithm [48] to compute the shortest path between each pair of stations based on the randomly generated incidence matrix. This ensures that the presumed characteristics of our test network will be realistic, as it was supposed that all vehicles always follow the shortest path to their destinations.

Based on a realistic PRT case [36], a $\lambda$ demand rate between five and 30 passengers per hour was generated for each specific station. This was programmed using the Poisson process provided in the Anylogic simulation software. This will ensure relatively realistic test cases for our four strategies. We generated 20 test scenarios based on this method. The test scenarios are of three hours each in order to represent specific periods of a day such as morning or afternoon time window. Consequently, the demand within each test scenario is considered to be stable and homogenous.

To capture and study different fleet sizes, we considered fleets of 100, 200, 300, and 400 PRT vehicles. The battery for each vehicle lasts 40 minutes, which represents $11 \%$ of the duration of the test (three hours). In our case, PRT batteries could be charged in the depot. We should note that there are other strategies to charge batteries of PRT other in the depot such as in PRT stations. That means the battery is being charged while empty vehicles are stationary in the stations. However, this option offers a disadvantage of increasing the infrastructure costs. Deciding to implement charging of vehicles within stations is related to the tactical and strategic level of decisions which is not the main scope of this paper. However, it could be implemented within our simulator by updating the battery status of each vehicles before departing from a station within the embarking active object.

To obtain computational results, we used tools provided by Anylogic. We used different dataset objects to store each of the total energy used, the wasted energy, the effective energy for each vehicle, and the wait time and the total time spent in the system for each passenger. Anylogic dataset objects are capable of storing data variables of type double. This object can also provide the minimum and maximum of all stored items, thus ensuring high flexibility. Anylogic statistical objects were used for the final statistics related to our simulation. The objects are capable of computing statistical information like the mean, minimum, maximum, and so forth from a dataset object. These objects were used to provide the final results for each test case.

Note that, for the FNVS strategy, we set the minimum number of vehicles in each station to two.

6.2. Test Results. Table 4 lists the results for each strategy and fleet size. These show the average waiting times, percentage of wasted energy, and the effective energy. 
TABLE 4: Summary of the results of different strategies.

\begin{tabular}{|c|c|c|c|c|}
\hline Strategy & Statistics & Empty movement $\%$ & Effective movement $\%$ & Mean waiting time (Min) \\
\hline \multirow{4}{*}{ FIFO } & Mean & 62.599 & 37.401 & 7 \\
\hline & Minimum & 56.414 & 32.909 & 4 \\
\hline & Maximum & 67.091 & 43.586 & 30 \\
\hline & Standard deviation & 3.022 & 3.022 & 6 \\
\hline \multirow{4}{*}{$\mathrm{NN}$} & Mean & 52.117 & 47.883 & 5 \\
\hline & Minimum & 45.588 & 40.075 & 2 \\
\hline & Maximum & 59.925 & 54.412 & 27 \\
\hline & Standard deviation & 3.260 & 3.260 & 6 \\
\hline \multirow{4}{*}{ FNVS } & Mean & 55.270 & 44.730 & 5 \\
\hline & Minimum & 48.423 & 40.273 & 1 \\
\hline & Maximum & 59.727 & 51.577 & 29 \\
\hline & Standard deviation & 2.483 & 2.483 & 6 \\
\hline \multirow{4}{*}{ WG } & Mean & 50.9254 & 49.0746 & 5 \\
\hline & Minimum & 40.4776 & 42.4659 & 1 \\
\hline & Maximum & 57.5341 & 59.5224 & 24 \\
\hline & Standard deviation & 4.2106 & 4.2106 & 6 \\
\hline
\end{tabular}

The goal of these strategies is to improve overall performance in terms of the quality of service and energy consumption. We can see that the different strategies succeeded to varying degrees in attaining these objectives. The results presented in Table 4 suggest that the strategic allocation of empty vehicles contributes considerably to improving system performance. We note that increasing the fleet size significantly improves quality of service. Using the first in, first out (FIFO) strategy, the wait time with a fleet size of 100 was 16 min. This was reduced by $12 \mathrm{~min}$ to $4 \mathrm{~min}$ by increasing the fleet size to 200 vehicles. Therefore, our first conclusion from these tests concerns the importance of fleet size for the quality of service in a PRT system. This is generally related to the on-demand aspect of this kind of system, in addition to the characteristic of privacy. Each trip requires one vehicle, contrary to mass transit transportation systems where many passengers take the same vehicle.

6.3. Analysis of Results. In this section, we discuss and analyze the quality of our proposed strategies through an analysis of the empty displacement of vehicles as well as the passenger wait times. We first conducted additional statistical analysis (statistical analysis was conducted using the GraphPad Prism statistical software; for more details, please see: http://www .graphpad.com/scientific-software/prism/) on the results.

In general, the objective of using statistical methods is to prove the superiority of one method over another. In fact, comparisons between simple means and between distributions can yield quite different conclusions. More specifically, a general statistical analysis is needed to ensure that the obtained results are meaningful. There are several statistical methods from the literature that can be used in our study [49]. In this paper, we used the nonparametric Friedman statistical test followed by Dunn's multiple comparisons test (nonexpert readers are referred to [49] for statistical analysis of Algorithms and to [50] for details about statistical tests). The use of the nonparametric test was motivated by the fact that it imposes fewer general conditions than parametric tests. This is especially true of the normality condition imposed on parametric tests. The Friedman statistical test was used because we have more than two sets of data. Therefore, the use of statistical methods will strengthen our comparative study between our different strategies (detailed results of our statistical analysis are provided in https://goo.gl/vBSiE5).

The WG strategy dominates all other strategies, excluding $\mathrm{NN}$, in terms of managing the empty displacement of vehicles. The results for these strategies can be explained by the fact that vehicles will remain in a station for as long as possible, and there is therefore a good chance that a passenger will find a waiting vehicle at a station. This feature is enhanced and extended in the FNVS strategy, as it determines a fixed number of vehicles to be available at each station. Consequently, there will be more empty displacement in the system. However, all stations will have empty vehicles available for service, contrary to the WG strategy where many empty vehicles can be at one station while other stations may lack vehicles.

Surprisingly, the NN strategy performs well with regard to managing the empty displacement of vehicles. This can be explained by the simple and effective rule used by this strategy. By sending the vehicle closest to the passenger who has waited the longest in order to service him/her, it manages the displacement of empty vehicles and passenger wait times in a relatively efficient manner.

Focusing on Figures 13-16, we can see the significant difference in waiting times for passengers between different stations in the WG strategy. This can be explained by the fact that due to passenger demand, more vehicles were located at some stations than others. Therefore, this strategy results in stations that are avoided by passengers because of the long waiting time.

The results also show a relative tradeoff between the energy used by empty vehicles and the wait times for passengers. 


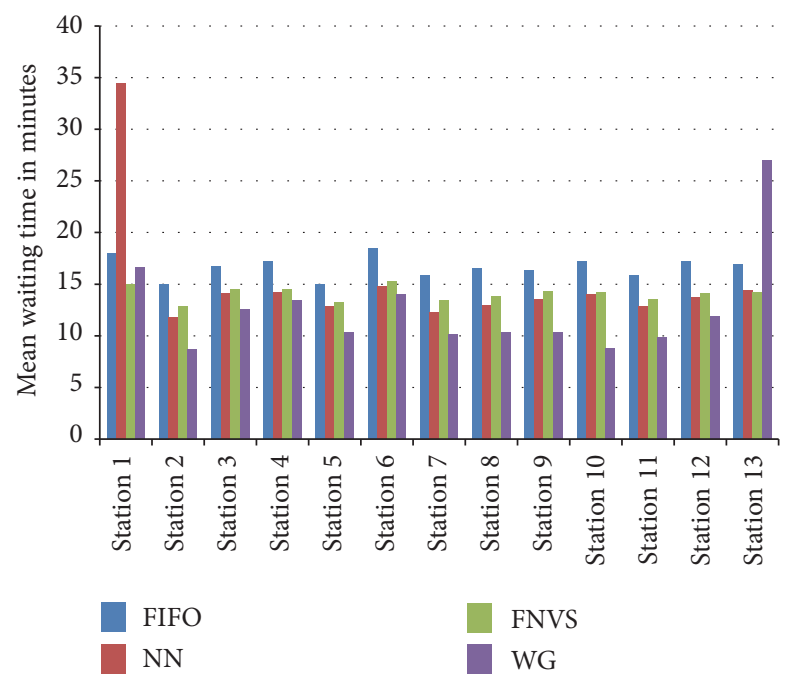

FIGURE 13: Wait time for each station with 100 vehicles.

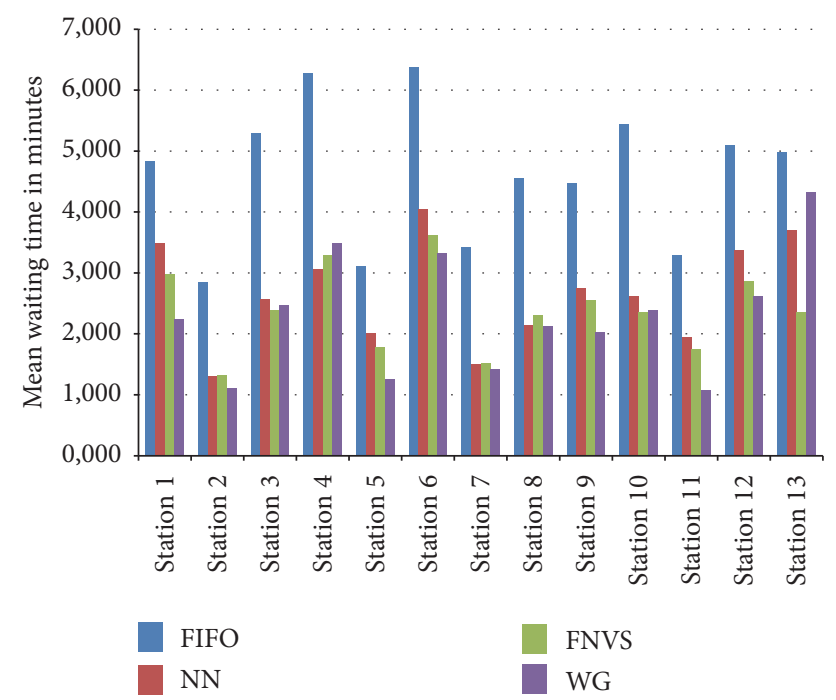

FIGURE 14: Wait time for each station with 200 vehicles.

Note that strategies that prioritize the choice of a closer station for the passenger are able to operate with lower energy consumption and empty vehicle movement. On the other hand, strategies that prioritize service quality (such as FVNS) are able to offer a high-quality service but consume more energy. However, the fact of having more vehicles would result in a high level of empty vehicle movements as empty pods would need to be rerouted in several cases. Therefore and as stated in the previous section, controlling the flow of empty movements was of a high interest for PRT system.

The number of available vehicles also influences service quality. The more vehicles there are, the better the service will be. However, this requires a significant initial investment for the PRT system. Fortunately, the energy savings and the increase in service quality may justify such an investment.

It should be noted that the difference in energy consumption due to the increase in fleet size could be controlled by optimizing the PRT fleet size. Jie et al. [51] have presented a

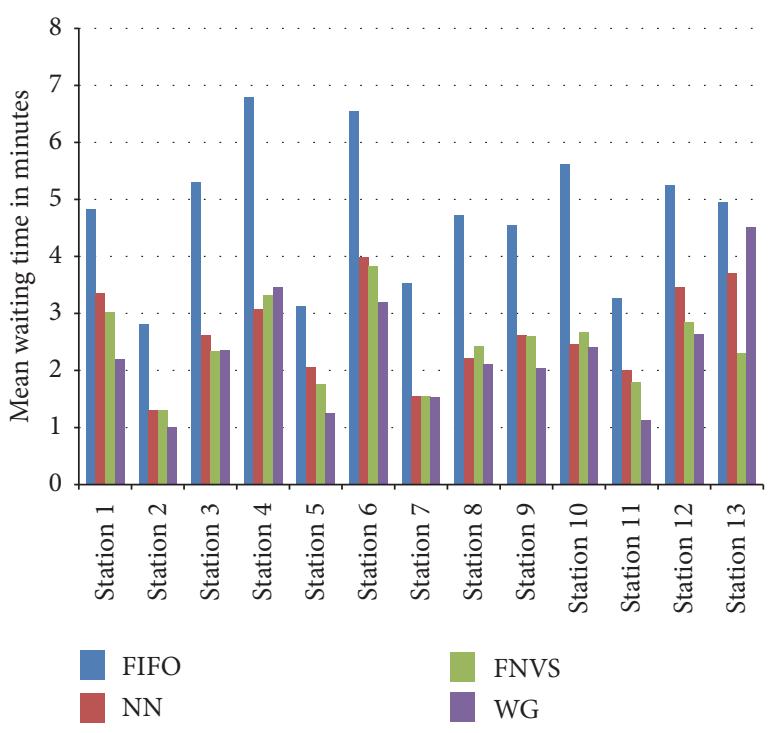

FIGURE 15: Wait time for each station with 300 vehicles.

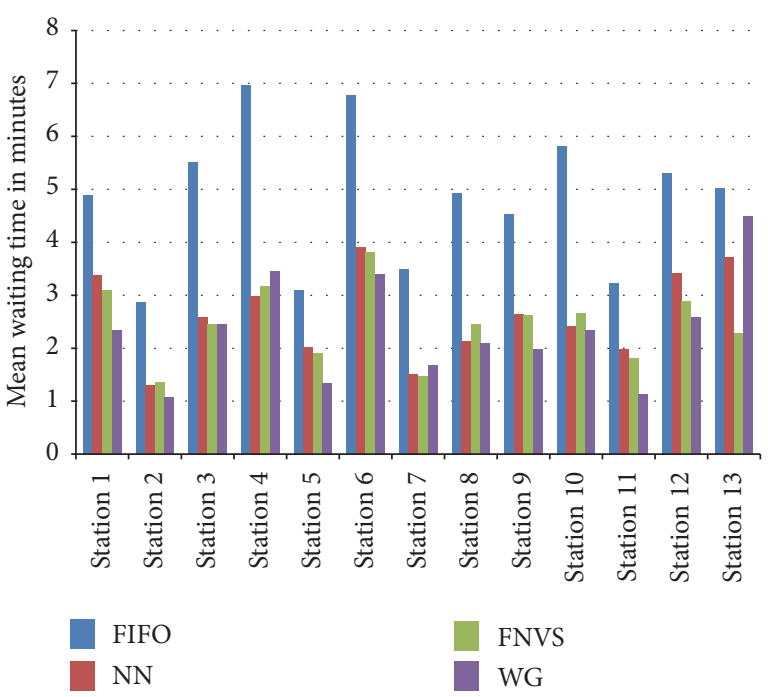

FIGURE 16: Wait time for each station with 400 vehicles.

framework for determining the fleet size of a PRT system, and their work can be extended to determine the optimal fleet size for our case.

6.4. Performance of the PRT System Based on Demand Spikes. In real time conditions and during specific events, an increase in the demand of passengers in different stations could be observed. In fact, stations located at a stadium, a convention center, or a hotel could observe at specific periods a drastic increase in the demand of transportation requests. Therefore, in this section, we wanted to test the effect of an increase in the demand in different specific stations on the PRT system. These tests would help us to get managerial insights of the system and to test the flexibility of our implemented simulation model. 


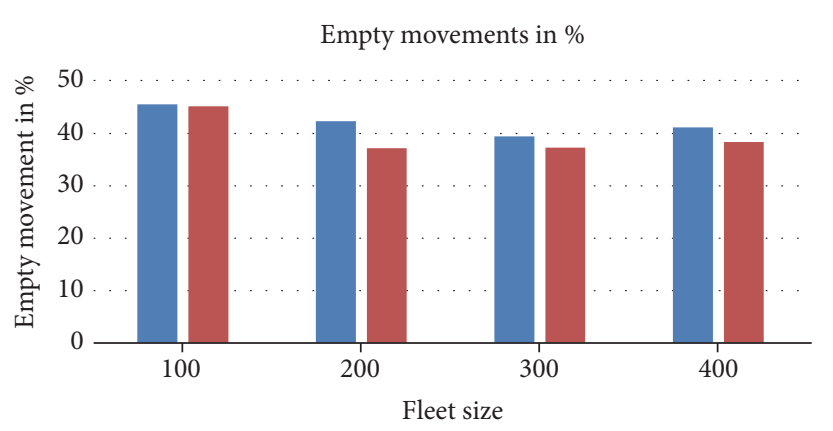

- Demand spikes

- Normal

(a) Evaluation of the empty vehicle movement in \% for each fleet size

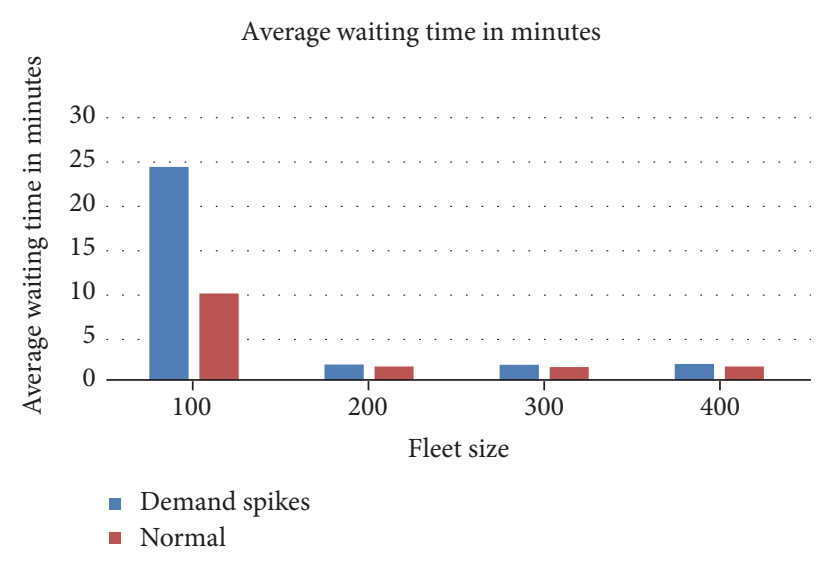

(b) Evaluation of the average waiting time in minutes for each fleet size

FIGURE 17: Pictures of the analysis of the performance of the PRT in the case of an increase of transportation demand.

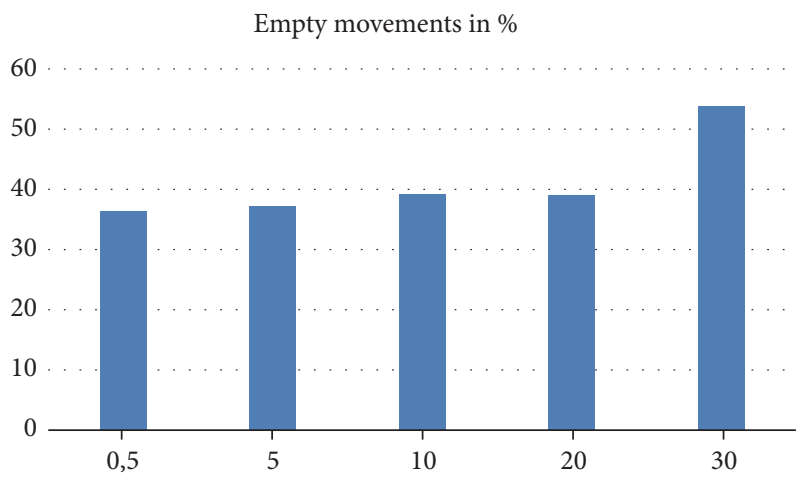

(a) Evaluation of the empty vehicle movement in \% for each charging time

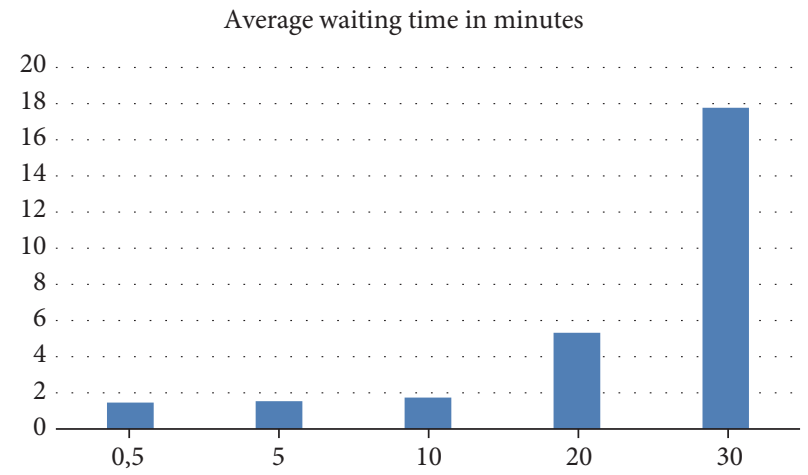

(b) Evaluation of the Average waiting time in minutes for each charging time

FIGURE 18: Pictures of the analysis of the charging time.

For this purpose, we choose a specific scenario while using the WG strategy and varying the fleet size between 100 and 400 vehicles. In the same scenario, we increased the transportation demand by double in four different stations. Results of this analysis are presented in Figure 17. The baseline scenarios are hereafter called the normal one and the scenario observing an increase in the passengers demands in different stations is called demand spikes.

The average passenger waiting time increased by $104 \%$ from the normal case in demand spike scenarios. We observed also an increase by $6 \%$ in the empty movements in the system during periods of an increase of the passengers demands.

Figure 17 shows the effect of the increase of the fleet size on handling the increase of the passengers demand in the PRT system. In fact, increasing the fleet size by 100 vehicles would reduce the average waiting time of passengers by 22 minutes from the 100 fleet size scenario.

In general, we could observe that the PRT system is able to meet peak demands in different stations while offering a satisfactory transportation service. This could be done while carefully adjusting the different parameters related to the PRT system such as the fleet size.
6.5. Performance of the PRT System Based on the Total Charging Time. In this section, we analyze the performance of the PRT system in the case of a using different technologies to recharge the electric batteries of vehicles. In fact, based on the used technologies, charging time of vehicles in the depot could vary between 30 minutes and 0.5 minute (installing a super supercapacitor would have an effect of reducing the total charging time to 30 seconds; for more details, the reader is refereed to [3]) [52,53]. Consequently, potential gain in system efficiency could be reached based on the charging strategy adopted.

In this set of experiments and based on a single scenario while using 200 PRT vehicles and the WG strategy, we proposed to test the effect of 5 different charging times (30 minutes, 20 minutes, 10 minutes, 5 minutes, and 0.5 minute) on the waiting time of passengers and the empty vehicle movements.

Results are presented in Figure 18.

The results show that an improvement in terms of service quality and empty movements could be observed while reducing the charging time of vehicles. In fact, reducing the charging time by 10 minutes from 30 to 20 minutes helps the system to gain a total of 12 minutes in terms of waiting 
time of passengers and $14 \%$ in terms of empty movements of vehicles. Also, using supercapacitor helps the system to reduce waiting time of passengers while having a small empty vehicle movements.

Generally, one could note that reducing the charging time of vehicles in the charging location reduces the vehicles unavailability due to charging operations. Consequently, smaller fleet size could be used for the system. This feature would help for the reduction in the initial investment as well as maintenance costs for PRT system as fewer vehicles would be needed. However, this potential gain could be mitigated as a high investment would be needed for installing supercapacitors.

\section{Conclusions and Future Works}

The PRT system is a relatively new electric transportation tool that has the potential to compete with prevailing modes of private transport. The main goal of this study was to propose a DSS and different dispatching strategies for the empty vehicle management in a PRT system. First, a valid lower bound on mean waiting time of passenger was developed based on a static deterministic routing problem. A mathematical formulation was proposed integrating several real-world constraints such as time window, battery, and distance constraints. This lower bound provided insights about the performance of the system in terms of transportation service.

Next, a DSS which integrates different dispatching strategies was proposed. It is based on the full integration of the management strategies for the PRT system. Also, four different proactive and reactive real time strategies to manage the movement of PRT vehicles were developed. A simulation model using the Anylogic simulation software was implemented as a part of our DSS. This platform makes it possible to analyze the behavior of different real time management strategies for PRT systems. Our simulations showed that the WG and NN strategies are the most energy efficient, whereas WG exhibits the best performance in terms of passenger wait times.

There is much scope for future works.

By way of extending this research, it would be interesting to implement other dispatching strategies for the management of PRT systems and to use a multi-agent-based simulation technique to offer more decentralized options for vehicle dispatching programs.

Specific Decomposition method for the proposed mathematical formulation could be proposed. In fact, the mathematical formulation was solved using IBM-Cplex. However, only small instances were solved by IBM-Cplex. Other more sophisticated methods such as Benders Decomposition, Column generation, or branch and price could be developed.

Uncertain travel times can also be implemented and incorporated into our simulation model. In fact and in PRT systems, empty vehicles movement affects congestion on the PRT network which affects the total travel time between each pair of stations. Consequently, travel time between PRT stations would depend heavily on the period of the day and the demand of passengers. Developing strategies that take into account this uncertain and dynamic version of the PRT system would be of a high interest and could be subject for future works. Finally, taking into account different shapes of the PRT network as a multiple depot topology could also be subject for future works.

\section{Conflicts of Interest}

The authors declare that there are no conflicts of interest regarding the publication of this paper.

\section{References}

[1] W. D. Cottrell, "Critical review of the personal rapid transit literature," in Proceedings of the 10th International Conference on Automated People Movers, vol. 174, p. 40, ASCE, 2005.

[2] H. William Merritt, Reflections on the New Systems Study Project, 1999, http://faculty.washington.edu/jbs/itrans/reflec2.htm.

[3] K. Mueller and S. P. Sgouridis, "Simulation-based analysis of personal rapid transit systems: Service and energy performance assessment of the Masdar City PRT case," Journal of Advanced Transportation, vol. 45, no. 4, pp. 252-270, 2011.

[4] J.-M. Won, K.-M. Lee, J. S. Lee, and F. Karray, "Guideway network design of personal rapid transit system: a multiobjective genetic algorithm approach," in Proceedings of the IEEE Congress on Evolutionary Computation (CEC '06), pp. 2215-2221, July 2006.

[5] J.-M. Won, H. Choe, and F. Karray, "Optimal design of personal rapid transit," in Proceedings of the IEEE Intelligent Transportation Systems Conference (ITSC '06), pp. 1489-1494, IEEE, September 2006.

[6] L. Jie, Y. S. Chen, H. Li, I. Andreasson, and H. van Zuylen, "Optimizing the fleet size of a personal rapid transit system: a case study in Port of Rotterdam," in Proceedings of the 13th International IEEE Conference on Intelligent Transportation Systems (ITSC '10), pp. 301-305, IEEE, Funchal, Portugal, September 2010.

[7] J. D. Lees-Miller and R. E. Wilson, "Sampling of redistribution of empty vehicles for personal rapid transit," Transportation Research Record, no. 2216, pp. 174-181, 2011.

[8] W. B. Daszczuk, W. Choromański, J. Miesćicki, and W. Grabski, "Empty vehicles management as a method for reducing passenger waiting time in Personal Rapid Transit networks," IET Intelligent Transport Systems, vol. 9, no. 3, pp. 231-239, 2015.

[9] W. B. Daszczuk, J. Mieścicki, and W. Grabski, "Distributed algorithm for empty vehicles management in personal rapid transit (PRT) network," Journal of Advanced Transportation, vol. 50, no. 4, pp. 608-629, 2016.

[10] O. Chebbi and J. Chaouachi, "Modeling on-demand transit transportation system using an agent-based approach," in Computer Information Systems and Industrial Management, pp. 316326, Springer, 2015.

[11] E. Fatnassi, O. Chebbi, and J. C. Siala, "Evaluation of different vehicle management strategies for the personal rapid transit system," in Proceedings of the 5th International Conference on Modeling, Simulation and Applied Optimization (ICMSAO '13), pp. 1-5, IEEE, Hammamet, Tunisia, April 2013.

[12] O. Chebbi and J. Chaouachi, "A decentralized management approach for on-demand transit transportation system," in Proceedings of the Second International Afro-European Conference for Industrial Advancement AECIA 2015. Advances in Intelligent 
Systems and Computing, vol. 427, pp. 175-184, Springer International Publishing, Cham, Switzerland, 2016.

[13] H. Zheng and S. Peeta, "Network design for personal rapid transit under transit-oriented development," Transportation Research Part C: Emerging Technologies, vol. 55, pp. 351-362, 2015.

[14] O. Chebbi and J. Chaouachi, "Optimal fleet sizing of personal rapid transit system," in Proceedings of the IFIP International Conference on Computer Information Systems and Industrial Management, pp. 327-338, Springer, Warsaw, Poland, September 2015.

[15] M. Mrad and L. Hidri, "Optimal consumed electric energy while sequencing vehicle trips in a personal rapid transit transportation system," Computers and Industrial Engineering, vol. 79, pp. 1-9, 2015.

[16] E. Fatnassi, O. Chebbi, and J. C. Siala, "Comparison of two mathematical formulations for the offline routing of personal rapid transit system vehicles," in Proceedings of the 19th International Conference on Methods and Models in Automation and Robotics (MMAR '14), pp. 554-559, September 2014.

[17] E. Fatnassi, O. Chebbi, and J. Chaouachi, "Discrete honeybee mating optimization algorithm for the routing of batteryoperated automated guidance electric vehicles in personal rapid transit systems," Swarm and Evolutionary Computation, vol. 26, pp. 35-49, 2016.

[18] O. Chebbi and J. Chaouachi, "Reducing the wasted transportation capacity of Personal Rapid Transit systems: an integrated model and multi-objective optimization approach," Transportation Research Part E: Logistics and Transportation Review, vol. 89, pp. 236-258, 2016.

[19] E. Fatnassi, J. Chaouachi, and W. Klibi, "Planning and operating a shared goods and passengers on-demand rapid transit system for sustainable city-logistics," Transportation Research Part B: Methodological, vol. 81, pp. 440-460, 2015.

[20] J. Edward Anderson, Some Lessons from the History of Personal Rapid Transit (PRT), 1996, http://faculty.washington.edu/jbs/ itrans/history.htm.

[21] C. Xithalis, "Synchronous control method for personal rapid transit systems," in Proceedings of the 10th International Conference on Application of Advanced Technologies in Transportation, Athens, Greece, May 2008.

[22] I. Andreasson, "Vehicle distribution in large personal rapid transit systems," Transportation Research Record, no. 1451, pp. 95-99, 1994.

[23] G. Göran Tegnér, M. Hunhammar, I. Andréasson, J.-E. Nowacki, and K. Dahlström, "PRT in Sweden: from feasibility studies to public awareness," in Proceedings of the 11th International Conference on Automated People Movers, Vienna, Austria, April 2007.

[24] J. B. Schneider, "Designing a high performance prt network for an edge city," 1997.

[25] J. Edward Anderson, Some History of PRT Simulation Programs, January 2007.

[26] J. D. Lees-Miller and R. Eddie Wilson, "Sampling for personal rapid transit empty vehicle redistribution," Transportation Research Record, vol. 2216, pp. 174-181, 2011.

[27] J. D. Lees-Miller, "Minimising average passenger waiting time in personal rapid transit systems," Annals of Operations Research, vol. 236, no. 2, pp. 405-424, 2016.

[28] V. Pillac, M. Gendreau, C. Gueret, and A. L. Medaglia, "A review of dynamic vehicle routing problems," European Journal of Operational Research, vol. 225, no. 1, pp. 1-11, 2013.
[29] I. Andreasson, "PRT as a supplement to existing transportation modes," in Proceedings of the 3rd International Conference on Urban Public Transportation Systems, pp. 10-22, November 2013.

[30] M. Mrad, O. Chebbi, M. Labidi, and M. A. Louly, "Synchronous routing for personal rapid transit pods," Journal of Applied Mathematics, vol. 2014, Article ID 623849, 8 pages, 2014.

[31] G. Berbeglia, J.-F. Cordeau, and G. Laporte, "Dynamic pickup and delivery problems," European Journal of Operational Research, vol. 202, no. 1, pp. 8-15, 2010.

[32] J. D. Lees-Miller and R. E. Wilson, "Proactive empty vehicle redistribution for personal rapid transit and taxis," Transportation Planning and Technology, vol. 35, no. 1, pp. 17-30, 2012.

[33] P. Toth and D. Vigo, Eds., The Vehicle Routing Problem, vol. 9 of SIAM Monographs on Discrete Mathematics and Applications, SIAM, Philadelphia, Pa, USA, 2002.

[34] S. Almoustafa, Distance-constrained vehicle routing problem: exact and approximate solution [Ph.D. thesis], Brunel University, 2013.

[35] S. Almoustafa, S. Hanafi, and N. Mladenovic, "Multistart branch and bound for large asymmetric distance-constrained vehicle routing problem," in Optimization Theory, Decision Making, and Operations Research Applications, A. Migdalas, A. Sifaleras, C. K. Georgiadis, J. Papathanasiou, and E. Stiakakis, Eds., vol. 31 of Springer Proceedings in Mathematics \& Statistics, pp. 15-38, Springer, New York, NY, USA, 2013.

[36] P. Teychenne and P. H. Bly, "Three financial and socio-economic assessments of a personal rapid transit system," in Proceedings of the 10th International Conference on Automated People Movers, chapter 32, pp. 1-16, Orlando, Fla, USA, 2005.

[37] Ç. Koç, T. Bektaş, O. Jabali, and G. Laporte, "The impact of depot location, fleet composition and routing on emissions in city logistics," Transportation Research Part B: Methodological, vol. 84, pp. 81-102, 2016.

[38] Ç. Koç, T. Bektaş, O. Jabali, and G. Laporte, “The fleet size and mix location-routing problem with time windows: formulations and a heuristic algorithm," European Journal of Operational Research, vol. 248, no. 1, pp. 33-51, 2016.

[39] A. Y. Chen, F. Peña-Mora, and Y. Ouyang, "A collaborative GIS framework to support equipment distribution for civil engineering disaster response operations," Automation in Construction, vol. 20, no. 5, pp. 637-648, 2011.

[40] J. E. Anderson, "Control of personal rapid transit systems," Journal of Advanced Transportation, vol. 32, no. 1, pp. 57-74, 1998.

[41] J. Banks, J. S. Carson, and B. L. Nelson, Discrete-Event System Simulation, Prentice-Hall International Series in Industrial and Systems Engineering, Prentice Hall, 1996.

[42] P. Lynch, K. Adendorff, V. S. S. Yadavalli, and O. Adetunji, "Optimal spares and preventive maintenance frequencies for constrained industrial systems," Computers and Industrial Engineering, vol. 65, no. 3, pp. 378-387, 2013.

[43] L. Huang, T. Chen, Y. Wang, and H. Yuan, "Congestion detection of pedestrians using the velocity entropy: a case study of Love Parade 2010 disaster," Physica A: Statistical Mechanics and Its Applications, vol. 440, pp. 200-209, 2015.

[44] G. Li, Z. Bie, Y. Kou, J. Jiang, and M. Bettinelli, "Reliability evaluation of integrated energy systems based on smart agent communication," Applied Energy, vol. 167, pp. 397-406, 2016.

[45] A. Borshchev, Y. Karpov, and V. Kharitonov, "Distributed simulation of hybrid systems with AnyLogic and HLA," Future Generation Computer Systems, vol. 18, no. 6, pp. 829-839, 2002. 
[46] K. K. Thakur, J. Sanchez, D. Hurnik, Z. Poljak, S. Opps, and C. W. Revie, "Development of a network based model to simulate the between-farm transmission of the porcine reproductive and respiratory syndrome virus," Veterinary Microbiology, vol. 180, no. 3-4, pp. 212-222, 2015.

[47] O. Chebbi and J. C. Siala, "Priority rule strategy for managing a Personal Rapid Transit system," in Proceedings of the 17th IEEE International Conference on Intelligent Transportation Systems (ITSC '14), pp. 2852-2857, October 2014.

[48] S. Hougardy, "The Floyd-Warshall algorithm on graphs with negative cycles," Information Processing Letters, vol. 110, no. 89, pp. 279-281, 2010.

[49] E. Alba, Parallel Metaheuristics: A New Class of Algorithms, Wiley Series on Parallel and Distributed Computing, WileyInterscience, Hoboken, NJ, USA, 2005.

[50] D. J. Sheskin, Handbook of Parametric and Nonparametric Statistical Procedures, CRC Press, Boca Raton, Fla, USA, 2003.

[51] L. Jie, C. Y. Sen, L. Hao, I. Andreasson, and H. J. Van Zuylen, "Optimizing the fleet size of a personal rapid transit system: a case study in port of Rotterdam," in Proceedings of the 13th International IEEE Conference on Intelligent Transportation Systems (ITSC '10), pp. 301-305, September 2010.

[52] Z. Ma, D. S. Callaway, and I. A. Hiskens, "Decentralized charging control of large populations of plug-in electric vehicles," IEEE Transactions on Control Systems Technology, vol. 21, no. 1, pp. 67-78, 2013.

[53] A. Gusrialdi, Z. Qu, and M. A. Simaan, "Scheduling and cooperative control of electric vehicles' charging at highway service stations," in Proceedings of the 53rd IEEE Annual Conference on Decision and Control (CDC '14), pp. 6465-6471, December 2014. 


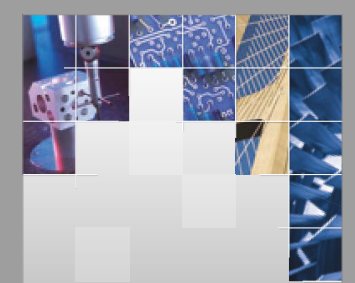

\section{Enfincering}
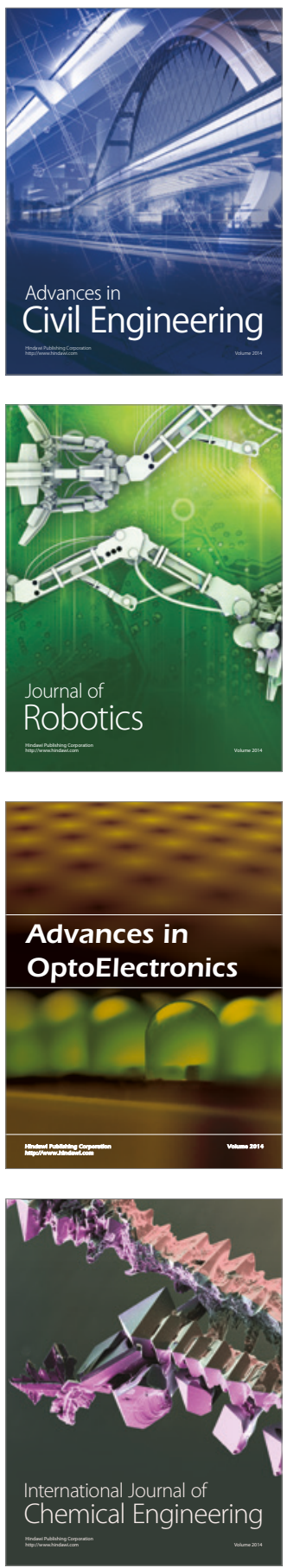

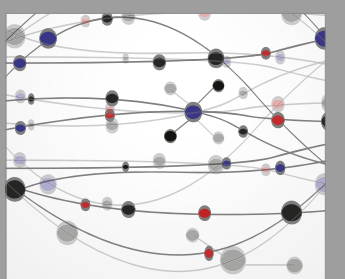

The Scientific World Journal

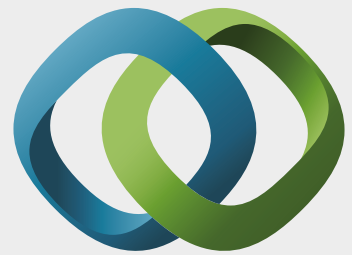

\section{Hindawi}

Submit your manuscripts at

https://www.hindawi.com
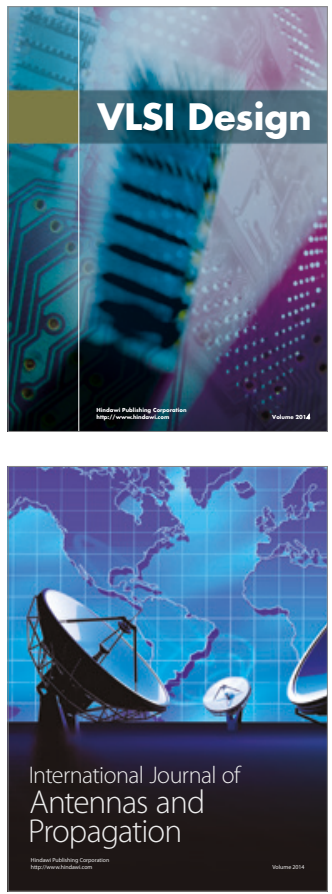

\section{Rotating}

Machinery
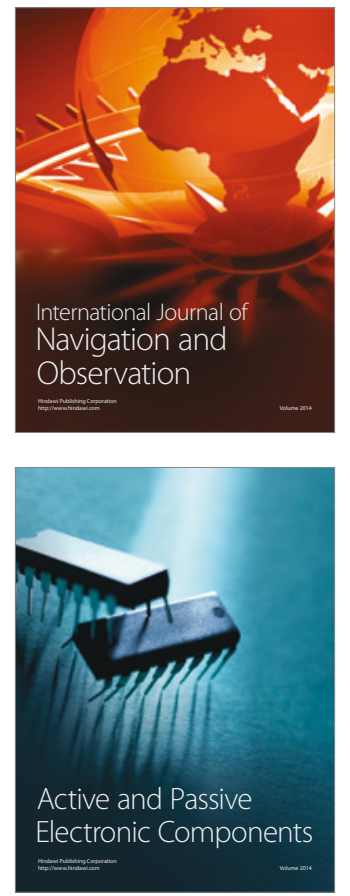
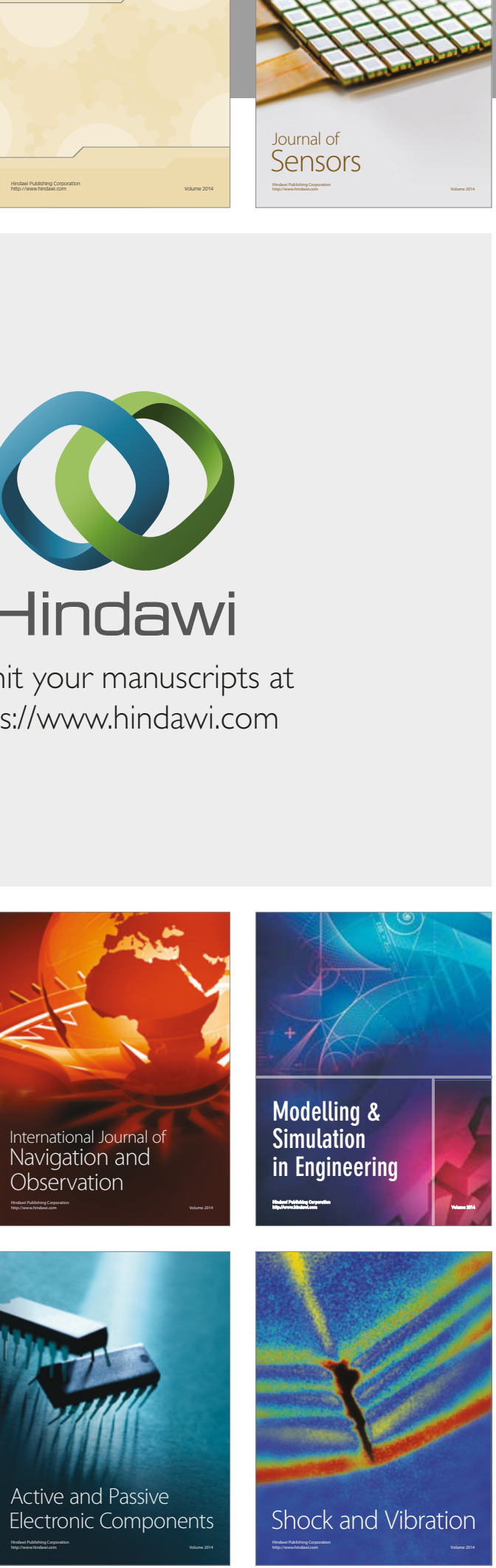
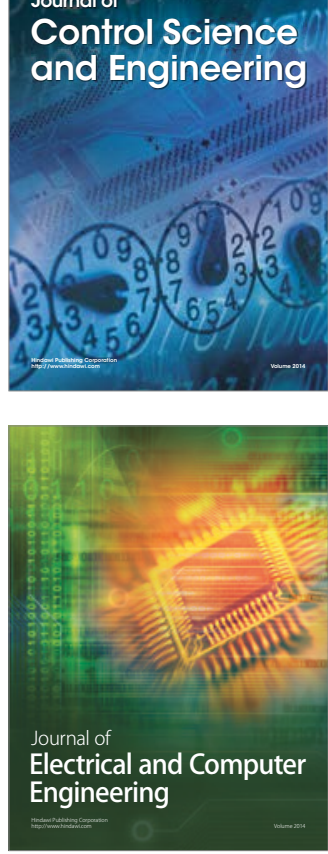

Distributed

Journal of

Control Science

and Engineering
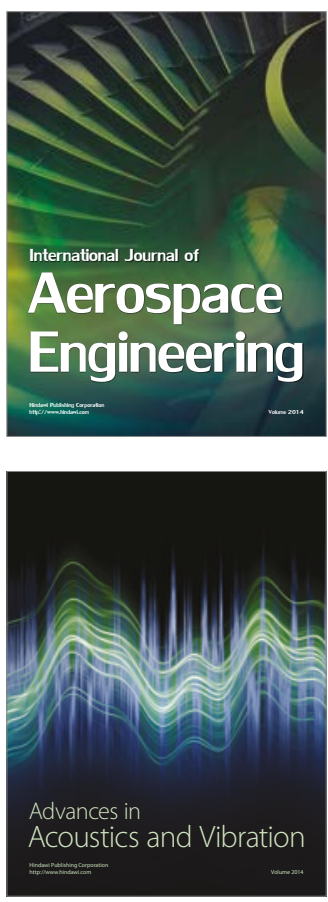

Sensor Networks 